\title{
Stability of falling liquid films on flexible substrates
}

\author{
J. Paul Alexander ${ }^{1}$, Toby L. Kirk ${ }^{2}$ and Demetrios T. Papageorgiou ${ }^{1} \dagger$ \\ ${ }^{1}$ Department of Mathematics, Imperial College London, London SW7 2AZ, UK \\ ${ }^{2}$ Mathematical Institute, Oxford University, Woodstock Road, Oxford, OX2 6GG, UK
}

(Received $\mathrm{xx}$; revised $\mathrm{xx}$; accepted $\mathrm{xx}$ )

The linear stability of a liquid film falling down an inclined flexible plane under the influence of gravity is investigated using analytical and computational techniques. A general model for the flexible substrate is used leading to a modified Orr-Sommerfeld problem addressed numerically using a Chebyshev tau decomposition. Asymptotic limits of long waves and small Reynolds numbers are addressed analytically and linked to the computations. For long waves, the flexibility has a destabilising effect, where the critical Reynolds number decreases with decreasing stiffness, even destabilising Stokes flow for sufficiently small stiffness. To pursue this further, a Stokes flow approximation was considered, which confirmed the long wave results, but also revealed a short wave instability not captured by the long-wave expansions. Increasing the surface tension has little effect on these instabilities and so they were characterised as wall modes. Wider exploration revealed mode switching in the dispersion relation, with the wall and surface mode swapping characteristics for higher wavenumbers. The zero Reynolds number results demonstrate that the long wave limit is not sufficient to determine instabilities so the numerical solution for arbitrary wavenumbers was sought. A Chebyshev tau spectral method was implemented and verified against analytical solutions. Short wave wall instabilities persist at larger Reynolds numbers and destabilisation of all Reynolds numbers is achievable by increasing the wall flexibility, however increasing the stiffness reverts back to the rigid wall limit. An energy decomposition analysis is presented and used to identify the salient instability mechanisms and link them to their physical origin.

\section{Introduction}

The dynamics of thin films have intrigued scientists for the past half century owing to their appearance in many daily life settings and their relevance to a wide variety of industrial and biophysical applications. In particular, films falling down an inclined flat plate under the influence of gravity, have received considerable attention since the experimental work of Kapitza \& Kapitza (1949) and Binny (1957), showed that they are accompanied by the development of large-amplitude surface waves. Many other applications of falling films arise in processes such as distillation and chemical reaction engineering, making the problem of fundamental interest.

Benjamin (1957) and Yih (1963), studied the hydrodynamic stability problem for falling films, and showed that the flow is unstable to long-wave surface modes that travel at twice the speed of the unperturbed flow at the interface. Instability is shown to require inertia (non-zero Reynolds number): for a given inclination angle $\beta$ to the horizontal there exists a critical Reynolds number, $R_{c}$ say, above which the flow is unstable; $R_{c}$ decreases to zero as $\beta \rightarrow \pi / 2$ when the incline becomes vertical. These linear surface

$\dagger$ Email address for correspondence: d.papageorgiou@imperial.ac.uk 
modes initially produce two-dimensional nonlinear waves whose structure is influenced inlet forcing as described in the experiments of Liu et al. (1995). A detailed computational study of the stability problem at arbitrary wavenumbers was carried out by Floryan et al. (1987); they focussed on small angles of inclination (at most 4 degrees) that require large critical Reynolds numbers for instability, and showed clearly the presence of an interfacial mode or surface mode, and a shear mode. Further down the plate the two-dimensional waves become susceptible to secondary instabilities to form three-dimensional waves Liu et al. (1995). For Reynolds numbers close to $R_{c}$, a nonlinear theory for long-waves was developed by Benney (1966) who took into account inertial, viscous, and capillary effects. At about the same time Shkadov (1967) used Polhausen approximation ideas originally applied to boundary layers, to derive a weighted residual model equation that compared favourably with experiments. In recent years such weighted residual nonlinear models have received considerable attention, e.g. Ruyer-Quil \& Manneville (2000); see also the monograph by Kalliadasis et al. (2012). It is also interesting to note that a weaklynonlinear simplification of the Benney equation results in the Kuramoto-Sivashinsky (KS) equation, see Sivashinsky \& Michelson (1980), that supports complex dynamics including spatiotemporal chaos.

Fluid flow over flexible surfaces has also been considered widely due to numerous physiological and technological applications. Of interest here is the work of Carpenter \& Garrad (1985) who consider spring-backed flexible plates (Kramer-type compliant surfaces) as a passive control mechanism of instabilities and transition to turbulence in boundary layers. Related studies for flows in channels with such compliant walls can be found in Gajjar \& Sibanda (1996), Davies \& Carpenter (1997), and a recent study of the effect of in-wall flexible blips on boundary layer flows, Pruessner \& Smith (2015). For multi-fluid flows Halpern \& Grotberg (1992) modelled the closure of liquid-coated pulmonary airways using lubrication theory and an elastic wall model that contains tension and damping. Flexible membrane models accounting for tension and damping (only a subset of the effects accounted for in spring-backed compliant surfaces) have been shown to enhance the rupture of thin films and destabilize the leading ridge of a thin liquid drop falling down a flexible incline Matar \& Kumar (2004, 2007).

Falling liquid film flows down flexible inclined substrates has been considered by several authors employing different models for the deformation of the substrate. The prevalent studies can be broadly categorized into two physical models for the elastic substrate: (i) the spring-backed plate membrane model of Carpenter \& Garrad (1985) used in the present study, and (ii) an elastic solid substrate of finite thickness over which the liquid film falls. For completeness we review the literature of elastic solid substrates and make connections to the present study. Elastic substrate models couple the elastic deformation field with the fluid flow and are typically characterized by the shear modulus of the material. The independent studies of Shankar \& Sahu (2006), and Gkanis \& Kumar (2006) were among the first to consider a Newtonian fluid falling film on a deformable solid layer. At first glance, the conclusions between the two studies appear inconsistent. Shankar \& Sahu (2006) conclude that the presence of the solid can stabilise the flow completely for ranges of given Reynolds number and inclination angle; indeed, their long wave analysis predicts that at zero Reynolds number the applied strain (the ratio of viscous to elastic forces) only acts to stabilise the flow, even though finite wavenumber instabilities are possible. On the other hand, Gkanis \& Kumar (2006) start with inertialess falling films that are stable when flowing over flat rigid substrates, and show that elasticity can destabilize the flow for both linear elastic as well as neo-Hookean nonlinear models. The reason for the differences are due to the models considered; Shankar \& Sahu (2006) assume a linear viscoelastic model for elasticity where the deviatoric part of the elastic 
stress tensor includes a term proportional to $\mu_{s} \partial_{t}\left(\partial u_{i} / \partial x_{j}+\partial u_{j} / \partial x_{i}\right)$ where $\mu_{s}$ is the viscosity of the solid, and this term is responsible for the different stability characteristics. Such models were used extensively in different setups involving falling films, including the presence of multiple solid layers Sahu \& Shankar (2016) and depth-dependent shear modulus in the elastic medium Mandloi \& Shankar (2020), as well as studies utilizing neo-Hookean substrates to model soft elastomeric coatings, Baingne \& Sharma (2019, 2020), Gaurav \& Shankar (2007, 2010), Jain \& Shankar (2007). A recent exposition of the different models and their evaluation regarding elastohydrodynamic instabilities in shear channel flows can be found in Patne \& Shankar (2019). Also of note is the weakly nonlinear study of Chokshi \& Kumaran (2008) who study high Reynolds number wall mode instabilities in Couette flows where one of the substrates is elastic and neo-Hookean, and the work of Thaokar et al. (2001) where a comparison is undertaken between Couette flow over a spring-backed plate model with that over a compressible viscoelastic membrane model. Of interest to our study is the spring-backed model in Thaokar et al. (2001) that is additionally allowed to extend tangentially via a phenomenological model linking tangential displacement linearly to the excess tangential stress of the fluid at the wall. It is found that in the absence of tangential motion (i.e. a plate model essentially identical to ours), the flow is stable in the low Reynolds number regime, at least for the range of parameters considered. Allowing tangential wall motion is found to induce instability even at zero Reynolds number and we expect analogous instabilities in the problem studied here also if tangential wall motion were allowed. Thaokar et al. (2001) also emphasize that at large Reynolds numbers the tangential wall motion is a higher order effect and does not affect the stability characteristics. In the present work we pick a Carpenter-type model without any tangential wall displacement in the linearized motion as has been done by numerous other authors as described next.

Simpler forms of the model used here were considered by Matar et al. (2007) who investigated the effect of substrate flexibility on the nonlinear long-wave regime in falling film flows. With a flexible membrane model similar to that used by Halpern \& Grotberg (1992) for flow in flexible tubes, they measured substrate flexibility through wall tension and damping terms. Using lubrication theory they then derived coupled Benneylike evolution equations for the film thickness and substrate deflection, followed by a weakly-nonlinear analysis that produced coupled equations analogous to the KuramotoSivashinsky equation. At higher Reynolds numbers, integral theory was used to derive extensions of the Shkadov equations Shkadov (1967). It was found that decreasing wall tension or damping promotes chaotic behaviour in the weakly-nonlinear regime (negligible inertia), and significant substrate deformations in the integral theory (significant inertia). In a follow-up study Sisoev et al. (2010) carried out additional computations and construction of nonlinear travelling waves, and their stability, that could be compared with experiments. Extensions of Matar et al. (2007); Sisoev et al. (2010) have also been carried out to include several other fluid dynamical effects, for example, viscoelasticity in the liquid film, and insoluble surfactants on the free surface, Peng et al. $(2014,2016)$.

The present work considers instabilities of falling films over compliant surfaces that account for more physical mechanisms than those studied to-date. We choose the general model of Carpenter \& Garrad (1985) that is representative of a large range of substrates due to the inclusion of inertia, rigidity, stiffness, tension and damping, and carry out a detailed theoretical investigation into the effect of each of these characteristics on the linear stability of arbitrary wavelength disturbances. Hence, our study extends that of Matar et al. (2007) who considered substrate tension and damping only, as well as a restriction to long waves, albeit nonlinear. As found in our computations the long wave assumption may not be a good one for certain wall parameters, with finite wavenumber 
disturbances being the only unstable modes beyond critical. Since the Reynolds numbers for falling films are lower than the aerodynamic ones considered in Carpenter \& Garrad (1985); Pruessner \& Smith (2015), for example, we derive the wall equation starting from the general nonlinear force balance and include the effects of viscous fluid traction that are neglected at high speed. In a way similar to that of Yih (1963), the linear stability is analysed by solving an Orr-Sommerfeld equation with modified boundary conditions due to the compliant wall.

Wall stiffness and damping are found to have the greatest influence on the stability of long waves. This is particularly interesting in view of previous studies that concentrated on long waves but with stiffness absent. The rigid wall limit can be approached in different ways, and particularly as either of the stiffness or damping parameters become large. In either limit, a falling film on a rigid wall at a given inclination angle $\beta$ is unstable to long waves above a critical Reynolds number. We find that starting with large stiffness and reducing it in turn decreases the critical Reynolds number. Below a certain stiffness the flow is unstable to all Reynolds numbers, even zero, providing a new type of inertialess instability absent for rigid walls. On the other hand starting with large damping and reducing it, once again introduces instability by reducing the critical Reynolds number. There is an intricate interplay between the relative sizes of stiffness and damping and we identify parameters for which the stability characteristics are very different from the rigid wall case.

Regarding the other wall parameters, i.e. inertia, tension and rigidity, we find that their effect is predominantly on short waves. In fact for ranges of parameters these shorter waves are found to dominate the instability. This emerges through mode crossing where, as parameters vary, the second stable mode dominates over the first long wave, an exchange of stabilities takes place and instability is supported at a finite wavenumber and a neighbouring band around it. We give several examples of such instabilities and emphasize that they cannot be captured with long wave theories. Interestingly, such instabilities were found to persist as the Reynolds number was decreased and also in the zero Reynolds number limit also described here.

The structure of the paper is as follows: in $\S 2$ we formulate the problem, and describe our flexible wall model (its derivation is included in the Appendix). In $\S 3$ we state the appropriate Orr-Sommerfeld boundary value problem and analyse the long-wave and Stokes flow limits in $\S 4$ and $\S 5$. In $\S 6$ we briefly describe the numerical methods and present code validations, and in $\S 6.2$ we present our results for arbitrary wavenumbers and extensive ranges of parameters. Section $\S 7$ is devoted to a discussion and conclusions.

\section{Formulation}

\subsection{Governing equations}

We shall consider the dynamics of an incompressible Newtonian fluid of viscosity $\mu$ and density $\rho$ falling under the influence of gravity down an infinitely long, flexible, impermeable surface inclined at an angle $\beta$ to the horizontal, shown in figure 1 . Above the film lies a passive, inviscid gas of constant pressure $p_{0}$, and the surface tension coefficient of the gas-liquid interface is denoted by $\sigma$. We use a rectangular coordinate system $(x, y, z)$ with $x$ measured down the slope (streamwise), $y$ measured in the transverse direction and $z$ measured normal to the slope. As we will exclusively deal with two-dimensional instabilities, we will neglect variation in the $y$ direction. Hence, the instantaneous substrate deflection from its equilibrium position $z=0$ at time $t$ is denoted by $z=\eta(x, t)$, and the instantaneous height of the gas-liquid interface relative to $z=0$ is denoted by $z=h(x, t)$. 


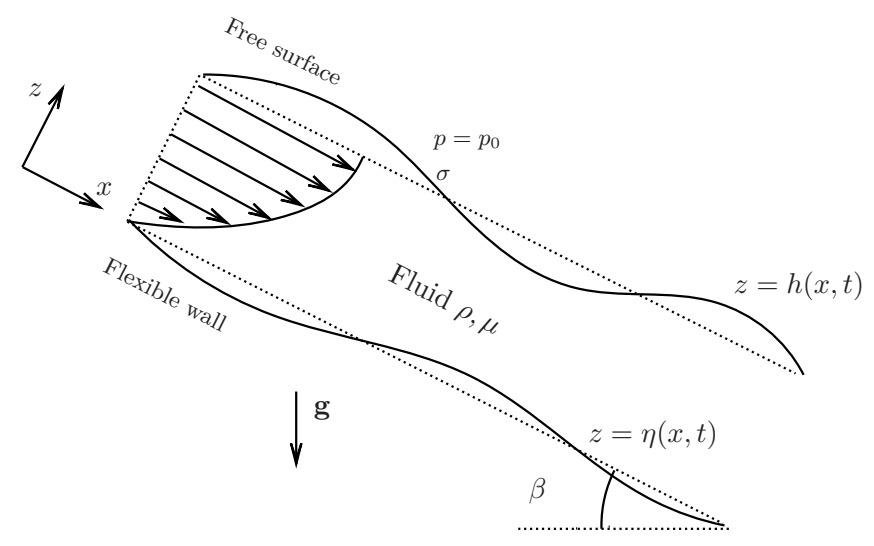

Figure 1: Schematic of the problem.

In this coordinate system, the velocity field in the liquid is $\boldsymbol{u}=(u(x, z, t), 0, v(x, z, t))$ with $u$ and $v$ the streamwise and normal velocity components, and acceleration due to gravity takes the form $\boldsymbol{g}=(g \sin \beta, 0,-g \cos \beta)$. The governing equations in the fluid are the two dimensional Navier-Stokes equations

$$
\begin{gathered}
u_{t}+u u_{x}+v u_{z}=-\frac{p_{x}}{\rho}+\frac{\mu}{\rho}\left(u_{x x}+u_{z z}\right)+g \sin \beta \\
v_{t}+u v_{x}+v v_{z}=-\frac{p_{z}}{\rho}+\frac{\mu}{\rho}\left(v_{x x}+v_{z z}\right)-g \cos \beta \\
u_{x}+v_{z}=0
\end{gathered}
$$

where $p$ is the fluid pressure and subscripts denote partial derivatives. The boundary conditions on the liquid air interface are the kinematic condition and normal and tangential stress balances, which can be written as

$$
\begin{aligned}
h_{t}+u h_{x} & =v \\
\left(1-h_{x}^{2}\right)\left(u_{z}+v_{x}\right)+4 h_{x} v_{z} & =0 \\
-\left(p-p_{0}\right)+2 \mu\left(\frac{1+h_{x}^{2}}{1-h_{x}^{2}}\right) v_{z} & =\frac{\sigma h_{x x}}{\left(1+h_{x}^{2}\right)^{3 / 2}},
\end{aligned}
$$

and it is understood that they are evaluated at $z=h(x, t)$. To arrive at these, the incompressibility condition $(2.1 c)$ has been used to simplify $(2.2 b)$, and subsequently $(2.2 b)$ to simplify $(2.2 c)$. These are the typical boundary conditions for a free surface with constant surface tension used for fluid films, and the derivation of which can be seen in, for example, Matar et al. (2007); Tseluiko \& Papageorgiou (2006).

Next we consider the flexible wall. The physical model we will use is shown schematically in figure 2, and was first described in Carpenter \& Garrad (1985) to model actual coatings used by Kramer (1957). To gain insight into the origin of the terms in a linear wall equation proposed by Carpenter \& Garrad (1985), and also to include the fluid's viscous traction (not present in Carpenter \& Garrad (1985)), we start with a nonlinear model that includes all the relevant physics and then simplify it using the reasonable assumption that longitudinal deflections are much smaller than transverse ones. This in turn provides a modified version of the linear wall equation used in Carpenter \& Garrad (1985). This derivation is included in Appendix A.

We will assume the surface of the compliant wall backing to be a two dimensional 


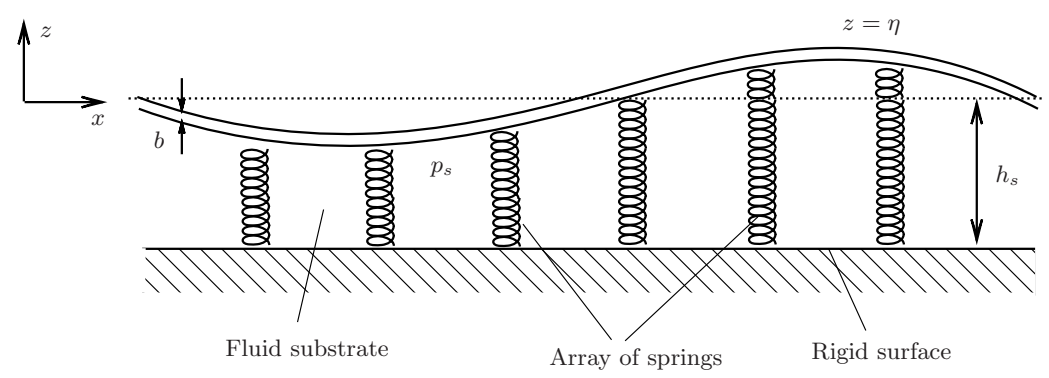

Figure 2: Schematic representation of the flexible wall model. An elastic sheet of thickness $b$ lies above a rigid surface and backed by an array of springs embedded in a fluid. The distance between springs is much smaller than any instability wavelength, enabling a continuum description.

inextensible elastic sheet of infinite length (in $x$ and $y$ directions), which is isotropic, and impermeable. Assume that it is thin enough for the longitudinal tension $T$ to be approximately constant across its thickness. It is supported a distance $h_{s}$ above a rigid surface by an array of springs each of stiffness $K$, with a fluid also backing the elastic plate, its motion unaffected by the presence of the springs. Provided the wavelengths of any instabilities considerably exceed the distance between the springs, we can assume the effect of the springs on the plate can be modelled as a continuous elastic foundation of stiffness $K$ for motion in the $z$ direction. We will also assume the presence of damping in the $z$ direction. The linear (in $\eta$ ) model equation we will use for the substrate deflection $\eta(x, t)$ is given by (see Appendix A)

$$
\rho_{w} b \eta_{t t}+D \eta_{t}-T \eta_{x x}+B \eta_{x x x x}+K \eta=p_{s}-p+2 \mu\left(v_{z}-\eta_{x}\left(u_{z}+v_{x}\right)\right),
$$

where $\rho_{w}$ is the plate density, $b$ is the plate thickness, $D$ is the damping coefficient, $T$ is the tension, $B$ is the flexural rigidity, $p_{s}$ is the substrate pressure on the back of the plate provided by the substrate fluid, and all fluid dynamical variables on the right hand side are evaluated on the substrate, $z=\eta(x, t)$. The case of a viscous and interactive fluid substrate can and has been considered just as in Carpenter \& Garrad (1985), but the two interesting limits of (i) zero substrate viscosity; and (ii) $h_{s} \ll$ (film thickness) can be shown to be equivalent to modifying the wall parameters $A_{D}$ and $A_{I}$ (which we define in $\S 2.2$ ) while keeping $p_{s}$ constant throughout. Thus for simplicity we shall assume $p_{s}$ is constant determined by the constant height solution, and corresponding to a passive substrate fluid.

Equation (2.3a) is almost identical to that of Carpenter \& Garrad (1985), except for the appearance of normal viscous wall traction from the fluid on the plate: the terms proportional to $\mu$ on the right hand side. The wall equations used by Halpern \& Grotberg (1992), and subsequently Matar et al. (2007); Matar \& Kumar (2004, 2007), retain this term but neglect bending stresses and wall inertia to retain just damping and tension on the left hand side (with no stiffness). For linear stability analysis, the equations of Matar et al. (2007); Matar \& Kumar $(2004,2007)$ are a special case of $(2.3 a)$ since for small deflections they are identical to $(2.3 a)$ but with $\rho_{w} b, B, K$ set to zero. The $\eta_{x}^{2}$ terms in their equations do not enter into their lubrication analysis anyway since they expand only up to $O(\delta)$ in the lubrication parameter, $\delta=$ film thickness/wavelength. Thus, for thin films, the wall equation above is an extension of the forms used in the literature Carpenter \& Garrad (1985); Matar et al. (2007); Matar \& Kumar (2004, 2007). 
In addition to $(2.3 a)$, the linearised form of the no-slip condition on the plate surface becomes

$$
u=0, \quad v=\eta_{t} \quad \text { on } \quad z=\eta .
$$

In practice, the material properties $\rho_{w} b$ (mass per unit area), $B$ (flexural rigidity), $T$ (tension), and $D$ (damping coefficient) are not all independent, but related, among other quantities, via the Young's modulus, E, and the Poisson ratio, $\nu$, of the plate material. The flexural rigidity is given by

$$
B=\frac{E b^{3}}{12\left(1-\nu^{2}\right)} .
$$

The damping coefficient can be determined from the damping ratio, $\zeta$, via the relation

$$
D=2 \zeta \sqrt{K \rho_{w} b}
$$

If required, actual values of the above quantities would have to be determined experimentally or found in materials tables.

The steady base flow of the system $(2.1 a)-(2.1 c)$ along with the conditions $(2.2 a)$ $(2.3 b)$, with constant film thickness $h_{0}$ and no substrate deflection, $\eta=0$, is given by (bars denote base state quantities)

$$
\begin{gathered}
\bar{u}=\frac{g \sin \beta}{2 \nu} z\left(2 h_{0}-z\right), \quad \bar{v}=0, \\
\bar{h}=h_{0}, \quad \bar{\eta}=0, \\
\bar{p}=p_{0}+\rho g \cos \beta\left(h_{0}-z\right), \quad \bar{p}_{s}=p_{0}+\rho g h_{0} \cos \beta .
\end{gathered}
$$

\subsection{Non-dimensionalisation of governing equations}

Our non-dimensionalisation is identical to that of Floryan et al. (1987), with velocities scaled by the Nusselt value $u_{m}=\rho g h_{0}^{2} \sin \beta / 2 \mu$, lengths with the film thickness $h_{0}$, time with $h_{0} / u_{m}$, and pressure fields with typical viscous stresses $\mu u_{m} / h_{0}$ (after a shift by $\left.p_{0}\right)$. Writing

$$
\begin{gathered}
x^{*}=\frac{x}{h_{0}}, \quad z^{*}=\frac{z}{h_{0}}, \quad t^{*}=\frac{t u_{m}}{h_{0}}, \quad h^{*}=\frac{h}{h_{0}}, \quad \eta^{*}=\frac{\eta}{h_{0}}, \\
u^{*}=\frac{u}{u_{m}}, \quad v^{*}=\frac{v}{u_{m}}, \quad p^{*}=\frac{\left(p-p_{0}\right) h_{0}}{\mu u_{m}}, \quad p_{s}^{*}=\frac{\left(p_{s}-p_{0}\right) h_{0}}{\mu u_{m}},
\end{gathered}
$$

casts the Navier-Stokes equations (2.1a)-(2.1c) into (after dropping stars)

$$
\begin{aligned}
R\left(u_{t}+u u_{x}+v u_{z}\right) & =-p_{x}+u_{x x}+u_{z z}+2, \\
R\left(v_{t}+u v_{x}+v v_{z}\right) & =-p_{z}+v_{x x}+v_{z z}-2 \cot \beta, \\
u_{x}+v_{z} & =0,
\end{aligned}
$$

where

$$
R=\rho u_{m} h_{0} / \mu,
$$

is the Reynolds number. Note that the viscous pressure scaling is appropriate for our study since the zero Reynolds number limit can be taken in a straightforward way. Conditions $(2.2 a)-(2.2 b)$ at the free surface $z=h$ are unchanged, while the normal stress balance equation $(2.2 c)$ becomes:

$$
-p+2\left(\frac{1+h_{x}^{2}}{1-h_{x}^{2}}\right) v_{z}=\frac{S h_{x x}}{\left(1+h_{x}^{2}\right)^{3 / 2}},
$$


where $S=\sigma / \mu u_{m}$ measures the ratio of capillary forces to inertial forces (it is an inverse Weber number). We note that the surface tension parameter $S$ can be written as $S=$ $K_{a} R^{-2 / 3}\left(\frac{3}{2} \sin \beta\right)^{-1 / 3}$ where $K_{a}=\left(3 \rho \sigma^{3} / g \mu^{4}\right)^{1 / 3}$ is the Kapitza number - see Floryan et al. (1987), for example - this is used in code-validation comparisons with Floryan et al. (1987).

The conditions on the wall $z=\eta$ become

$$
\begin{gathered}
u=0, \quad v=\eta_{t}, \\
A_{I} \eta_{t t}+A_{D} \eta_{t}-A_{T} \eta_{x x}+A_{B} \eta_{x x x x}+A_{K} \eta=p_{s}-p+2\left(v_{z}-\eta_{x}\left(u_{z}-v_{x}\right)\right),
\end{gathered}
$$

where five additional dimensionless groups emerge, $A_{I}, A_{D}, A_{T}, A_{B}, A_{K}$ and are defined by

$$
\begin{aligned}
& A_{I}=\frac{\text { wall inertia }}{\text { viscous stress }}=\frac{\rho_{w} b u_{m}^{2}}{h_{0}} \cdot \frac{h_{0}}{\mu u_{m}}=\rho_{w} b u_{m} / \mu, \\
& A_{D}=\frac{\text { damping }}{\text { viscous stress }}=D u_{m} \cdot \frac{h_{0}}{\mu u_{m}}=\frac{D h_{0}}{\mu}, \\
& A_{T}=\frac{\text { wall tension }}{\text { viscous stress }}=\frac{T}{h_{0}} \cdot \frac{h_{0}}{\mu u_{m}}=\frac{T}{\mu u_{m}}, \\
& A_{B}=\frac{\text { flexural rigidity }}{\text { viscous stress }}=\frac{B}{h_{0}^{3}} \cdot \frac{h_{0}}{\mu u_{m}}=\frac{B}{h_{0}^{2} \mu u_{m}}, \\
& A_{K}=\frac{\text { spring stiffness }}{\text { viscous stress }}=K h_{0} \cdot \frac{h_{0}}{\mu u_{m}}=\frac{K h_{0}^{2}}{\mu u_{m}} .
\end{aligned}
$$

Note that we have chosen the same time-scales for the fluid and wall equations in order to ensure the most general fluid-structure interaction. It then follows that in situations where the wall time-scales are much larger than the fluid ones, the parameter $A_{I}$ in our model will be small (indeed it can be set to zero). With the above scalings the dimensionless base flow becomes

$$
\begin{array}{cl}
\bar{u}=z(2-z), \quad \bar{v}=0, & \bar{h}=1, \quad \bar{\eta}=0, \\
\bar{p}=2 \cot \beta(1-z), & \bar{p}_{s}=2 \cot \beta .
\end{array}
$$

\section{Linear stability}

We linearise about the steady state (2.13) and look for normal mode solutions for the perturbations of the form $(\tilde{u}, \tilde{v}, \tilde{p}, \tilde{h}, \tilde{\eta})=(\hat{u}(z), \hat{v}(z), \hat{p}(z), \hat{h}, \hat{\eta}) \mathrm{e}^{\mathrm{i} \alpha(x-c t)}+c . c$., where tilde decorations denote the perturbations and $\alpha$ and $c$ are the non-dimensional wavenumber and phase velocity, respectively. Introducing a perturbation stream function $\tilde{\psi}=$ $\phi(z) \mathrm{e}^{\mathrm{i} \alpha(x-c t)}$, so that $\hat{u}=\phi^{\prime}(z), \hat{v}=-\mathrm{i} \alpha \phi(z)$ (primes denote $z$-derivatives) and eliminating the pressure leads to the Orr-Sommerfeld equation

$$
\left(\frac{d^{2}}{d z^{2}}-\alpha^{2}\right)^{2} \phi-i \alpha R(\bar{u}-c)\left(\frac{d^{2}}{d z^{2}}-\alpha^{2}\right) \phi+i \alpha R \bar{u}^{\prime \prime} \phi=0 .
$$

Working with the boundary conditions on the free-surface $(2.2 a),(2.2 b),(2.10)$, and eliminating $\hat{p}$ and $\hat{h}$ gives, now at $\underline{z=1}$ :

$$
\phi^{\prime \prime}+\left(\alpha^{2}+\frac{2}{1-c}\right) \phi=0
$$




$$
\mathrm{BC} 2 \quad i \phi^{\prime \prime \prime}+\left(\alpha R(1-c)-3 i \alpha^{2}\right) \phi^{\prime}-\left(\frac{2 \alpha \cot (\beta)+S \alpha^{3}}{1-c}\right) \phi=0 .
$$

A similar linearisation of $(2.11 a)-(2.11 b)$ at the elastic wall yields, now at $\underline{z=0}$ :

$$
\begin{gathered}
c \hat{\eta}=\phi, \\
\phi^{\prime}=-2 \hat{\eta}, \\
-\alpha^{2} c^{2} A_{I} \hat{\eta}-i \alpha c A_{D} \hat{\eta}+\alpha^{2} A_{T} \hat{\eta}+\alpha^{4} A_{B} \hat{\eta}+A_{K} \hat{\eta}=-\bar{p}^{\prime} \hat{\eta}-\hat{p}+2\left(-i \alpha \phi^{\prime}-i \alpha \hat{\eta} \bar{u}^{\prime}\right) .
\end{gathered}
$$

Finally, using (3.4) to eliminate $\hat{\eta}$, and the expression $\hat{p}=R\left[-(\bar{u}-c) \phi^{\prime}+\bar{u}^{\prime} \phi\right]+\left(\phi^{\prime \prime \prime}-\right.$ $\left.\alpha^{2} \phi^{\prime}\right) /(\mathrm{i} \alpha)$ from $(2.8 b)$, we obtain at $z=0$ :

$$
\text { BC3 } \quad \phi^{\prime}=-2 \phi / c,
$$

BC4 $\left(-\alpha^{2} c^{2} A_{I}-i \alpha c A_{D}+\alpha^{2} A_{T}+\alpha^{4} A_{B}+A_{K}-2 \cot (\beta)\right) \phi=-\frac{c}{i \alpha} \phi^{\prime \prime \prime}+2 i \alpha \phi$.

In the rest of the paper we will refer to conditions (3.2)-(3.3) and (3.7)-(3.8) as BC1, BC2, BC3 and BC4. The Orr-Sommerfeld equation (3.1) and BC1-BC2 are the same as for the case of a fluid film falling down a flat rigid incline, but instead of $\phi^{\prime}(0)=\phi(0)=0$ corresponding to $u=v=0$ at $z=0$ for a solid wall, flexibility gives rise to BC3 and the much more complex $\mathrm{BC} 4$ involving not only $R$ but also the five parameters $A_{I}, A_{D}, A_{T}, A_{B}, A_{K}$ characterizing the mechanical properties of the wall. Equation (3.1) with boundary conditions BC1-BC4 constitutes an eigenvalue problem for $c$ leading to a dispersion relation,

$$
c \equiv c\left(\alpha, R, \beta, S, A_{I}, A_{D}, A_{T}, A_{B}, A_{K}\right) .
$$

For temporal instability $\alpha$ is real and $c=c_{r}+\mathrm{i} c_{i}$ is complex; stability or instability follows for $c_{i}(\alpha)<0$ or $c_{i}(\alpha)>0$, respectively. Neutral curves, defined by $c_{i}=0$, in parameter spaces such as the $\alpha-R$ or $A_{K}-R$ planes are computed and discussed extensively. We determine such curves at arbitrary values of the parameters in order to identify the salient instability mechanisms. In general, this must be done numerically (see $\S 6.2$ ), but the limit of long-waves, $\alpha \ll 1$, considered next, is both informative and serves as a check for the numerical work.

\section{Asymptotic solution for long waves, $\alpha \ll 1$}

We follow Yih (1963) and look for a regular asymptotic expansion in powers of $\alpha$

$$
\phi(z ; \alpha)=\phi_{0}(z)+\alpha \phi_{1}(z)+\ldots, \quad c(\alpha)=c_{0}+\alpha c_{1}+\ldots,
$$

where the $\phi_{j}(z), c_{j}, j=0,1, \ldots$ are $\mathcal{O}(1)$ quantities. Such expansions are especially useful for order one Reynolds numbers, and readily provide the classical critical Reynolds number instability threshold $R>R_{c}=\frac{5}{4} \cot \beta$, in the rigid wall case Yih (1963). Here we use a similar analysis to address the flexible wall problem at hand. Substitution of (4.1) into (3.1) and BC1-BC4 and solving order by order in $\alpha$ gives, at $\mathcal{O}(1)$,

$$
\phi_{0}(z)=a_{2} z^{2}+a_{3} z-a_{3}, \quad c_{0}=2,
$$

with $a_{2}, a_{3}$ constants (normalization gives one unknown constant at leading order), and at $\mathcal{O}(\alpha)$ we find, after some algebra,

$$
c_{1}=\frac{8}{15} i R-\frac{2}{3} i \cot \beta-\frac{1}{3} i \tilde{S}+\frac{i(\tilde{S}+2 \cot \beta)^{2}}{3\left(\tilde{S}-4 \tilde{A}_{I}-2 i \tilde{A}_{D}+A_{K}+\tilde{A}_{T}+\tilde{A}_{B}\right)} .
$$



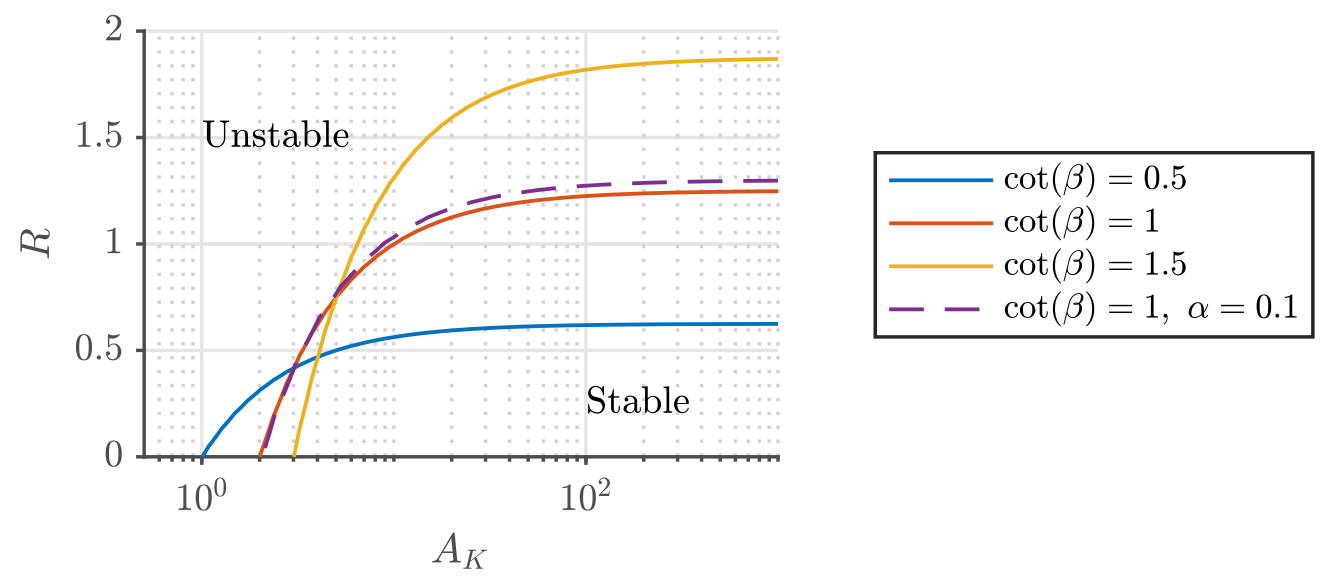

Figure 3: Neutral curves in the $R-A_{K}$ plane for various values of $\cot \beta$. Verification of the numerical solution is also given by the dashed line for $\alpha=0.1$, with the accuracy improving as $\alpha \rightarrow 0$.

The expression for $\phi_{1}(z)$ was deemed unilluminating and too long to include here. In (4.3) we have chosen to retain surface tension and all wall parameters and have defined tilde variables by

$$
\widetilde{S}:=\alpha^{2} S, \quad \widetilde{A}_{I}:=\alpha^{2} A_{I}, \quad \widetilde{A}_{D}:=\alpha A_{D}, \quad \widetilde{A}_{T}:=\alpha^{2} A_{T}, \quad \widetilde{A}_{B}:=\alpha^{4} A_{B} .
$$

If $S, A_{I}, A_{D}, A_{T}$ and $A_{B}$ are order one quantities, then their tilde equivalents are small. We can select some (if not all) tilde quantities to be of $\mathcal{O}(1)$ in order to evaluate their effect in the long wave limit. Note that the stiffness parameter $A_{K}$ survives without any rescaling in the long wave limit, and that the next most important effect is due to damping that can be retained if $\widetilde{A}_{D}=\mathcal{O}(1)$, i.e. $A_{D} \sim 1 / \alpha$. Note also that with the tilde quantities absent, the expansion (4.3) breaks down as $A_{K} \rightarrow 0$ and a separate asymptotic treatment is needed - see Section 4.3 .

The result (4.3) recovers the case studied by Matar et al. (2007) who considered large surface tension falling films on vertical plates that have no stiffness $\left(A_{K}=0\right)$ and asymptotically large values of damping and tension, i.e. $\widetilde{A}_{D}$ and $\widetilde{A}_{T}$ non-zero in our notation. Using these in (4.3) along with $\cot \beta=R=0$ we find $3 c_{1 i}=$ $-\left(4 \widetilde{S} \widetilde{A}_{D}^{2}+\widetilde{S}^{2} \widetilde{A}_{T}+\widetilde{S} \widetilde{A}_{T}^{2}\right) /\left[\left(\widetilde{S}+\widetilde{A}_{T}\right)^{2}+4 \widetilde{A}_{D}^{2}\right]<0$, and hence plate elasticity is stabilizing in this case as found by Matar et al. (2007).

\subsection{Wall parameters and surface tension of $\mathcal{O}(1)$}

In such cases $S$ and all tilde parameters in (4.3) tend to zero as $\alpha \rightarrow 0$, providing the purely imaginary quantity

$$
c_{1}=\left(\frac{8}{15} R-\frac{2}{3} \cot \beta+\frac{4}{3 A_{K}}(\cot \beta)^{2}\right) i .
$$

The stability boundary is given by

$$
R=R_{c}=\frac{5}{4} \cot \beta-\frac{5(\cot \beta)^{2}}{2 A_{K}},
$$

with instability if $R>R_{c}$. The only wall parameter present in this result is the stiffness $A_{K}$, and we see immediately that the rigid wall result emerges for $A_{K} \gg 1$, as expected. 

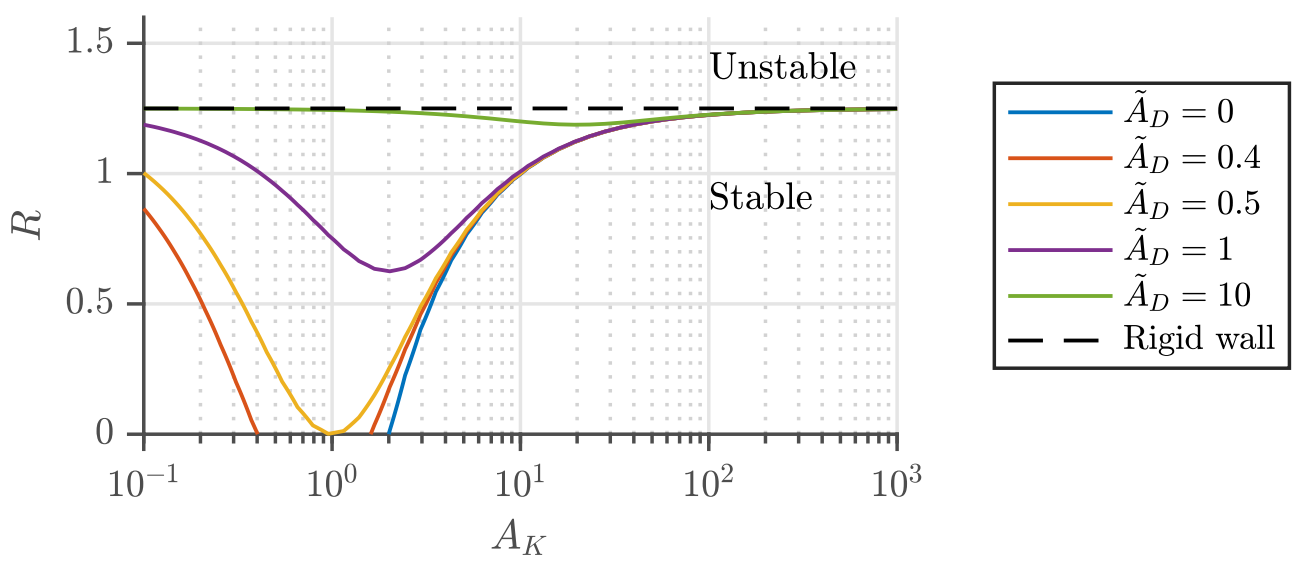

Figure 4: Neutral curves in the $A_{K}-R$ plane for various $\tilde{A}_{D}=\alpha A_{D}=O(1)$, with $\cot \beta=1, \tilde{S}=\tilde{A}_{I}=\tilde{A}_{T}=\tilde{A}_{B}=0$

The result (4.6) shows that stiffness induces long wave instability in the sense that for any finite $A_{K}$ and a given angle $\beta$, the critical Reynolds number $R_{c}$ decreases relative to that of a rigid wall. In addition, as $A_{K}$ decreases to values smaller than $A_{K}^{*}=2 \cot \beta$, we find instability for all $R$, including $R=0$. Figure 3 shows the neutral curves in the $R-A_{K}$ plane, where the critical stiffness $A_{K}^{*}=2 \cot \beta$ is located on the $R=0$ axis for a given $\beta$ as shown. The additional instability as $A_{K}$ is decreased is clearly seen. As the plate becomes vertical $\cot \beta \rightarrow 0$, hence the critical Reynolds number decreases to zero for every stiffness, however large, in agreement with the rigid wall case. The figure also includes a neutral curve computed from the Orr-Sommerfeld problem and shown by the dashed curve for the case $\alpha=0.1$ and $\cot \beta=1$. We see that agreement is very good, but this would of course deteriorate as $\alpha$ increases. Such computations are described later where it is seen that short waves can become important unlike the rigid wall case.

\subsection{Inclusion of damping, $\widetilde{A}_{D}=O(1)$}

Inspection of (4.3) and (4.4) shows that apart from $A_{K}$ which is of order one, the next largest effect is due to the damping, $\widetilde{A}_{D}=\alpha A_{D}$. Here we investigate its effect in the long wave regime by assuming $\widetilde{A}_{D}=\mathcal{O}(1)$ with all other tilde parameters set to zero. In a spring/damper system like the one modelled here, damping controls the dissipation of energy in the system. Underdamped systems oscillate and overdamped ones return to the base state monotonically; coupling this with the fluid dynamics and interfacial perturbations is useful in understanding the fluid-structure instability mechanisms. Mathematically, it is also seen from (4.3) that for $\alpha \ll 1$ the tension $\widetilde{A}_{T}$, rigidity $\widetilde{A}_{B}$ and inertia $\widetilde{A}_{I}$ just contribute to a renormalisation of the stiffness $A_{K}$, whereas the inclusion of damping implies that $c_{1 r} \neq 0$.

The effect of damping on neutral stability curves analogous to Figure 3 , is depicted in Figure 4 for fixed angle $\beta=\pi / 4$. Since $\widetilde{S}=\widetilde{A}_{I}=\widetilde{A}_{T}=\widetilde{A}_{B}=0$, equation (4.3) gives

$$
c_{1 i}=\frac{8}{15} R-\frac{2}{3} \cot \beta+\frac{4(\cot \beta)^{2} A_{K}}{3\left(A_{K}^{2}+4 \widetilde{A}_{D}^{2}\right)} .
$$

It is clear from (4.7) that $\widetilde{A}_{D}$ has a stabilizing effect, and interestingly an island of stability emerges for sufficiently small $R<(5 / 4) \cot \beta$ and $A_{K}$, as seen in Figure 4 
for $\widetilde{A}_{D}=0.4$. More precisely, setting $R=0$ in (4.7) shows that $c_{1 i}=0$ when $A_{K}=$ $\cot \beta \pm \sqrt{(\cot \beta)^{2}-4 \widetilde{A}_{D}^{2}}$, and as long as $\widetilde{A}_{D} \leqslant(1 / 2) \cot \beta$, there will be a region of inertialess instability when

$$
\cot \beta-\sqrt{(\cot \beta)^{2}-4 \widetilde{A}_{D}^{2}}<A_{K}<\cot \beta+\sqrt{(\cot \beta)^{2}-4 \widetilde{A}_{D}^{2}}
$$

This instability region disappears when $\widetilde{A}_{D}=(1 / 2) \cot \beta$, with the neutral curve having a local minimum at $A_{K}=\cot \beta, R=0$, as seen in Figure 4 for the case $\widetilde{A}_{D}=0.5$. As the damping increases, the neutral curve tends to that for a rigid wall for all values of $A_{K}$ as seen from (4.7) when $\widetilde{A}_{D} \gg 1$. It is worth noting that in the expression (4.3) the last term is due to wall flexibility, and hence the rigid wall result follows if any of the wall parameters become large.

\subsection{Zero stiffness, $A_{K}=0$}

Having $A_{K} \neq 0$ is critical for the validity of the expansion leading to (4.3); indeed if the other parameters are not scaled, then the asymptotic expansion breaks down as $A_{K} \rightarrow 0$. This limit is not uninteresting and is comparable to the model used in Matar et al. (2007), hence for completeness the alternative asymptotic solution with zero stiffness is included. Expanding as in (4.1), we now find that $c_{0}$ has a non-zero imaginary part (higher order terms need to be determined and solvability conditions imposed as before). After a lot of algebra we find

$$
c_{0}=1+\frac{1}{A_{D}} \pm \frac{\sqrt{\left(A_{D}-b\right)^{2}-\left(b^{2}-1\right)}}{A_{D}}, \quad \text { where } \quad b=1+\frac{2}{3}(\cot \beta)^{2}>1 .
$$

Inspection of the expression under the square root shows that $c_{0 i} \neq 0$ if $A_{D}$ satisfies

$$
b-\left(b^{2}-1\right)^{1 / 2}<A_{D}<b+\left(b^{2}-1\right) .
$$

It follows that as the plate becomes vertical and $b \rightarrow 1+$, admissible damping values get squeezed in a small region around $A_{D}=1$. On the other hand, as the plate becomes horizontal and $b \rightarrow \infty$, we have instability for all $A_{D}$. For the numerical examples where we typically take $\beta=\pi / 4$, the instability window is $\frac{1}{3}<A_{D}<3$.

\section{Solution in the zero Reynolds number limit}

When $R \rightarrow 0$ the Orr-Sommerfeld equation (3.1) reduces to

$$
\left(\frac{d^{2}}{d z^{2}}-\alpha^{2}\right)^{2} \phi=0
$$

and boundary condition (3.3) on $z=1$ becomes

$$
i \phi^{\prime \prime \prime}-3 i \alpha^{2} \phi^{\prime}-\left(2 \alpha \cot \beta+S \alpha^{3}\right) \frac{\phi}{1-c}=0 .
$$

The other boundary conditions (3.2), (3.7), (3.8) remain unchanged and we emphasize that all wall parameters and the surface tension parameter $S$ are order one quantities. The effects of stiffness $A_{K}$ and damping $A_{D}$ will be considered in detail below, all other parameters held fixed mostly at unit values. The solution of (5.1) is $\phi=A \sinh (\alpha z)+$ $B \cosh (\alpha z)+C \alpha z \sinh (\alpha z)+D \alpha z \cosh (\alpha z)$, where $A, B, C, D$ are constants to be found from the homogeneous boundary conditions (3.2), (5.2), (3.7), (3.8). Writing $\boldsymbol{X}=$ $(A, B, C, D)^{T}$, we have a matrix problem $M \boldsymbol{X}=0$ that has non-trivial solutions if 


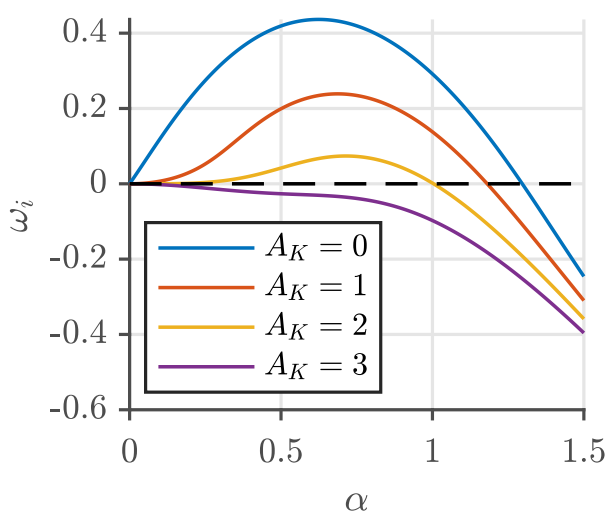

(a)

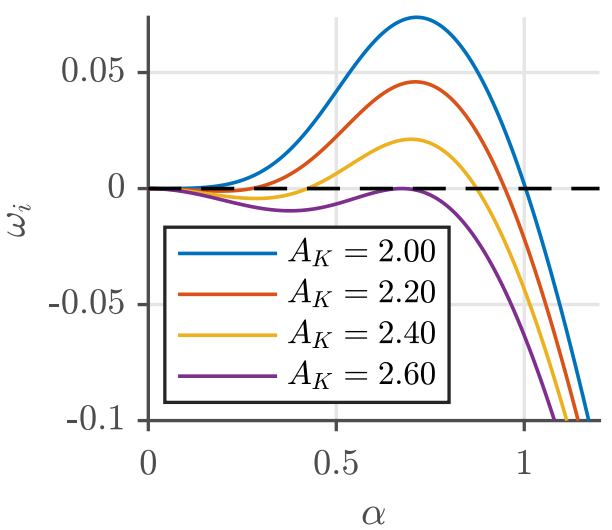

(b)

Figure 5: Growth rate curves for Stokes flow, for various $A_{K}$ indicated on the figure. The other parameters are taken to be $\cot \beta=S=A_{D}=A_{T}=A_{B}=A_{I}=1$.

$\operatorname{det} M=0$, leading to $P_{5}(c)=0$ where $P_{5}$ is a polynomial in $c$ of degree 5 . Two of the roots were introduced into the system when multiplying boundary conditions by $c$ and $1-c$, and are removed from the results; so $P_{5}(c)=c(1-c) P_{3}(c)$. The roots were found analytically using the Python package Sympy and in what follows we present stability results as crucial wall parameters such as the stiffness and damping are varied, with other parameters held fixed at unit values.

\subsection{Effect of stiffness, $A_{K}$}

The substrate stiffness is studied first as it was found to have the greatest effect among the wall parameters. The growth rate $\omega_{i}=\alpha c_{i}$ is shown in figure 5 for the choice of the other parameters $\cot \beta=S=A_{D}=A_{T}=A_{B}=A_{I}=1$. Figure 5a demonstrates that for $A_{K}$ below a critical value $A_{K c} \approx 2.60$ the flow is unstable, as predicted qualitatively, but not quantitatively as we shall see, by the long wave analysis. It also shows that for the special case of zero stiffness the gradient at the origin becomes non-zero and was found to match with the zero stiffness asymptotics of Section 4.3. In panel $5 \mathrm{~b}$ we show details of the growth rate in the interval $2<A_{K}<3$, and find that there is a finite wavenumber instability with $\alpha \approx 0.67$ (the wavelength is approximately 9 times the undisturbed film thickness) when $A_{K}$ just exceeds $A_{K c}$. This instability occurs before the long wave one at $\alpha=0$, and as a result the earlier long wave prediction $A_{K}^{*}=2 \cot \beta$ (yielding the value 2 in this case) gives a lower prediction for the critical $A_{K}$ calculated by considering all wavenumbers.

\subsection{Effect of damping, $A_{D}$}

Here we take $\cot \beta=S=A_{K}=A_{T}=A_{B}=A_{I}=1$ and consider the effect of $A_{D}$ on the stability. The results are shown in Figure 6 with $A_{D}$ varying between 0 and 2 . The overall trends are as expected with an increase in damping leading to flow stabilization. Note that the long wave analysis as well as results at $R=0$ (omitted for brevity), show that this stabilization is limited by the rigid wall case that derives in the limit $A_{D} \rightarrow \infty$. Conversely, decreasing the damping destabilises the flow as expected, but as seen in Figure 6 the critical wavenumber $\alpha$ below which instability is supported is weakly affected. Some interesting mode-crossing behaviour is also found at smaller $A_{D}$, for example the curves corresponding to $A_{D}=0$ and $A_{D}=0.2$ in Figure 6 . Looking at 


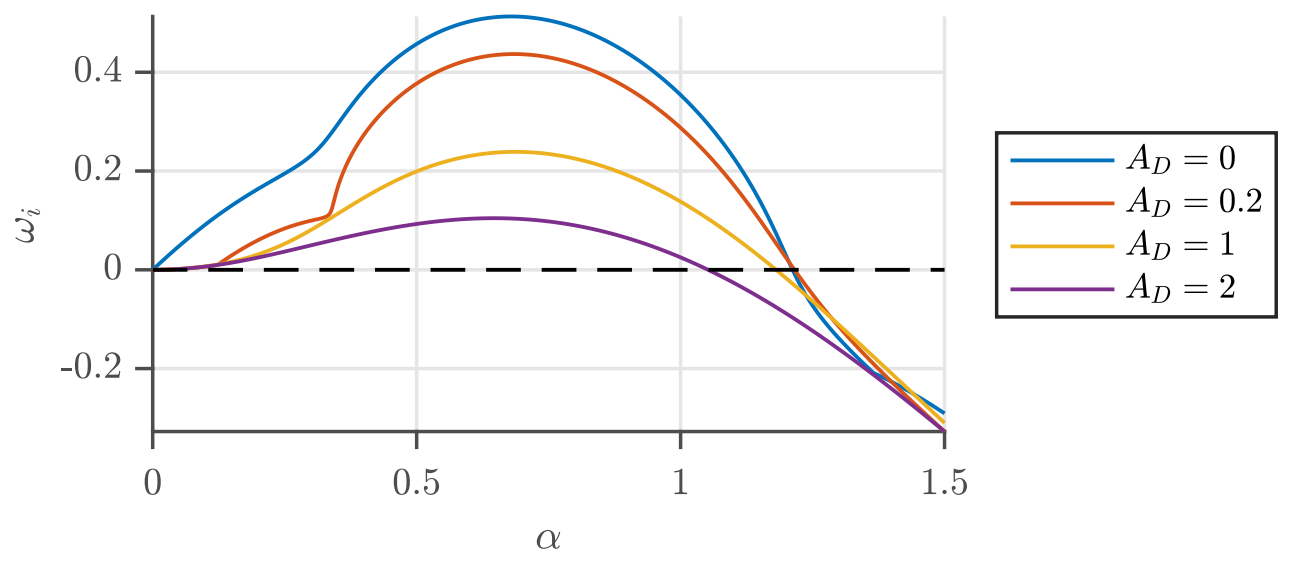

Figure 6: Growth rate curves for Stokes flow, for various $A_{D}$ indicated on the figure. The other parameters are $\cot \beta=S=A_{K}=A_{T}=A_{B}=A_{I}=1$. The curves for $A_{D}=0$ and $A_{D}=0.2$ indicate mode crossing at a finite $\alpha$.

$A_{D}=0.2$, for example, we observe a corner in the $\omega_{i}$ curve at $\alpha=\alpha_{c} \approx 0.35$ where two modes cross. Our computations produce results for multiple modes and plots such as 6 are constructed by tracking the largest value of $\omega_{i}$ as $\alpha$ varies. For $A_{D}=0.2$, long wave instability arises due to an interfacial mode, also termed first mode in the sequel, for $0<\alpha \lesssim \alpha_{c}$, and mode-switching takes place at $\alpha_{c}$ with a wall mode, also termed second mode, becoming more unstable for $\alpha \gtrsim \alpha_{c}$. The interfacial mode is characterized by being neutral at $\alpha=0$ and long-wave unstable - see below also. To clarify the switching of the most unstable mode from interfacial to wall mode, we plot them together for values of $A_{D}$ near 0.2 (all other parameters as in Figure 6), providing data for their growth rates and corresponding phase velocity (note that there is a countable infinity of further modes that are all stable and of no concern in this discussion). The results are shown in Figure 7 for $A_{D}=0.25,0.2225,0.2$ in panels $7 \mathrm{a}-\mathrm{c}$, respectively. The interfacial mode is shown in blue and passes through the origin, and the wall mode is shown in red. For the two larger values $A_{D}=0.25$ and 0.2225 , the second mode is unstable but has a smaller maximum growth rate than the first mode. In addition, the wavespeed of the second mode is always larger than that of the first mode as shown in panels $7 \mathrm{a}-\mathrm{b}$. The value $A_{D}=0.2225$ provides the damping that causes mode switching as we can see by comparison of plots $7 \mathrm{a}$ and $7 \mathrm{c}$ - here, the real and imaginary parts of $c$ are the same at the crossing point. Before swapping, the most unstable mode is also long wave unstable whereas after swapping the most unstable mode is long wave stable. It is also interesting to note that the wall mode appears to support larger phase velocities as $\alpha \rightarrow 0$, something that is analogous to gravity waves in the long wave limit, see Whitham (1999). These waves are always stable since $\omega_{i}<0$ for the second mode if $\alpha$ is below a critical value. The results for $A_{D}=0.2$ with $\alpha=0.3$, for instance, provide an interesting example where the most unstable mode travels much faster than the analogous rigid wall mode, something that could be useful in experiments. 


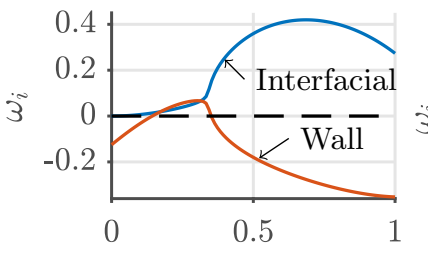

$\alpha$

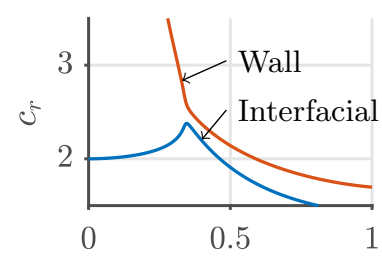

$\alpha$

(a) $A_{D}=0.25$
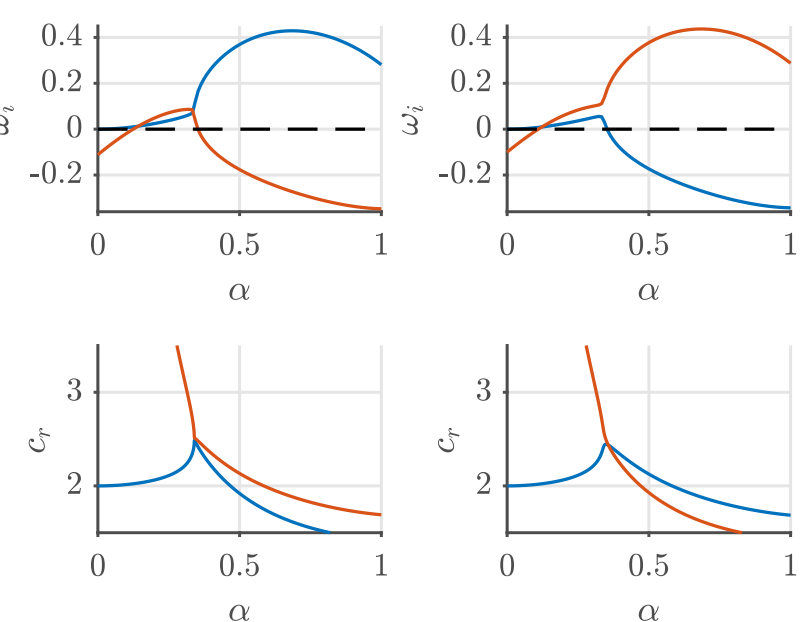

(b) $A_{D}=0.2225$

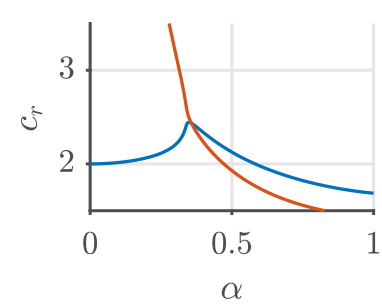

(c) $A_{D}=0.2$

Figure 7: Plots of the two most unstable modes: interfacial mode - blue, wall mode red. Top panels show the growth rates $\omega_{i}$ and bottom panels the corresponding phase velocity $c_{r}$, for $A_{D}=0.25$ (left), $A_{D}=0.2225$ (middle), and $A_{D}=0.2$ (right). The other parameters are $\cot \beta=S=A_{T}=A_{B}=A_{K}=A_{I}=1$.

\section{Stability characteristics at arbitrary Reynolds and wave numbers}

\subsection{Numerical methods and code validation}

Sections 4 and 5 produced asymptotic solutions for small $\alpha$ but arbitrary $R$, and small $R$ and arbitrary $\alpha$, respectively. The latter analysis indicates that in the presence of wall flexibility the maximally growing waves occur at order one wave numbers, hence it is important to extend our stability studies to arbitrary wave numbers and Reynolds numbers computationally. We briefly describe our numerical methods used to solve the Orr-Sommerfeld eigenvalue problem (3.1) subject to the interfacial conditions (3.2)-(3.3), and the wall conditions (3.7)-(3.8). The solution is approximated by a truncated series of Chebyshev polynomials of the first kind, in particular following the $D^{2}$ Chebyshev tau scheme of Dongarra et al. (1996). This involves introducing $\chi(z)=\left(\frac{\mathrm{d}^{2}}{\mathrm{~d} z^{2}}-\alpha^{2}\right) \phi(z)$, which transforms the single fourth order equation for $\phi$ into a pair of second order equations for $\phi$, and $\chi$. We write

$$
\phi(z)=\sum_{n=0}^{N+2} \phi_{n} T_{n}(z), \quad \chi(z)=\sum_{n=0}^{N+2} \chi_{n} T_{n}(z),
$$

where $T_{n}(z)$ is the $n$th Chebyshev polynomial, and $N$ is a measure of the truncation (with $N+3$ polynomials used). Differentiation is performed directly by operating on the $T_{n}$. Hence, substituting into the equations and BCs for $(\phi, \chi)$ and using the orthogonality properties of the $T_{n}$ Dongarra et al. (1996), we can write the system as a finitedimensional nonlinear eigenvalue problem for $c$ :

$$
\left(c^{2} \boldsymbol{B}_{2}+c \boldsymbol{B}_{1}+\boldsymbol{B}_{0}\right) \boldsymbol{x}=\mathbf{0} \quad \text { where } \quad \boldsymbol{x}=\left(\phi_{0}, \ldots, \phi_{N+2}, \chi_{0}, \ldots, \chi_{N+2}\right)^{T},
$$

and $\boldsymbol{B}_{2}, \boldsymbol{B}_{1}, \boldsymbol{B}_{0}$ are $2(N+3) \times 2(N+3)$ matrices with their bottom four rows representing the boundary conditions (3.2), (3.3), (3.7) and (3.8) in turn expressed in terms of the Chebyshev coefficients $\boldsymbol{x}$. Notice that we have a nonlinear $c^{2}$ term in $(6.2)$ which is absent 


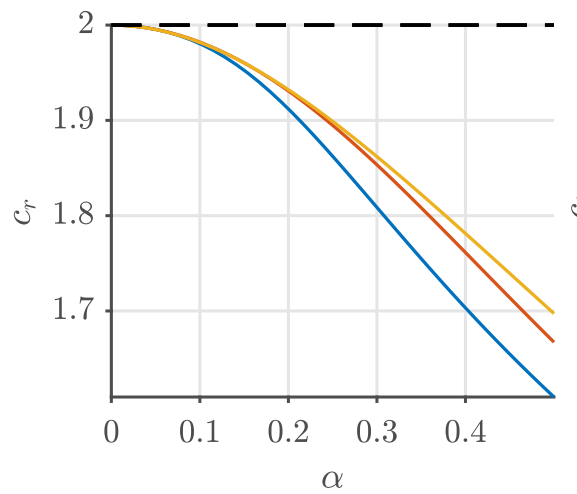

(a)

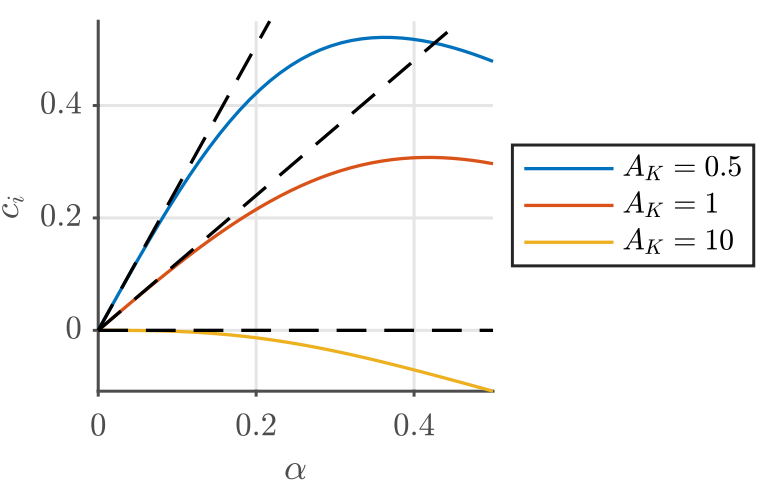

(b)

Figure 8: Comparison between computed values (solid lines) of $c_{r}$ and $c_{i}$ with the asymptotic long-wave solution (dashed lines), for various stiffnesses $A_{K}=0.5,1,10$ indicated on the figure; also, $R=1, A_{I}=A_{D}=A_{T}=A_{B}=1, N=50$ and $S=\cot \beta=1$.

for this method of discretisation for the case of flow down a rigid wall. This nonlinearity is due solely to the inertia of the flexible wall, i.e., the second order time derivative of $\eta$ in the equation for the wall deflection (2.11b). It appears only through (3.8) as the first term on the left hand side and is proportional to $A_{I}$, implying that the matrix $\boldsymbol{B}_{2}$ contains zeroes everywhere except for the last row, and $\boldsymbol{B}_{2}=\mathbf{0}$ if $A_{I}=0$. The eigenvalues are computed using the Matlab routines polyeig or eig when $A_{I} \neq 0$ or $A_{I}=0$, respectively. Spurious or infinite eigenvalues with unphysical growth rates arise due to the singular rows of the matrices $\boldsymbol{B}_{2}, \boldsymbol{B}_{1}$, Dawkins et al. (1998): half the rows of $\boldsymbol{B}_{1}$ are zero owing to the introduction of $\chi$, and another row is zero owing to boundary condition (3.7), as both $\chi$ and (3.7) are independent of $c$. We ignore these eigenvalues numerically, but the one due to (3.7) is removed entirely by elimination; see McFadden et al. (1990) for details. The numerical results were computed for zero Reynolds number and compared against the analytical results of section 5 for a variety of parameters, with excellent agreement.

The code was validated by comparing computed variations of $c_{r}$ and $c_{i}$ with $\alpha$, with their long wave counterparts presented in $\S 4$. For completeness, a small selection of these checks for values of the stiffness parameter $A_{K}=0.5,1,10$ are presented in Figure 8, with $\cot \beta, R$ and the other wall parameters set to unity. The computational results are depicted with solid curves and the corresponding long wave ones by the dashed straight lines. Agreement improves as $\alpha$ decreases, as expected, and the results indicate that long wave theory does a reasonable job for $\alpha \lesssim 0.1$, at least for this set of parameters. Lastly, the long-wave neutral curves in the $R-A_{K}$ plane that were analysed in $\S 4$ can be reproduced by the numerics for small $\alpha$. For instance in Figure 3 the computed neutral curve corresponding to $\cot \beta=1$ and $\alpha=0.1$ is seen to be in very good agreement with the long-wave neutral curve for the whole range of $R$ and $A_{K}$ considered.

\subsection{Numerical results}

We begin by presenting results for an inclination angle $\beta=\pi / 4$ for which the critical Reynolds number is 1.25. Smaller angles and large Reynolds numbers will be considered later. As with the analytical results, we start by varying the stiffness parameter $A_{K}$ with $A_{D}, A_{T}, A_{B}, A_{I}$ set to unity. Neutral stability curves in the $\alpha-R$ plane are given in Figures $9 \mathrm{a}$ and $9 \mathrm{~b}$ for surface tension parameters $S=1$ and $S=1000$, respectively, the 


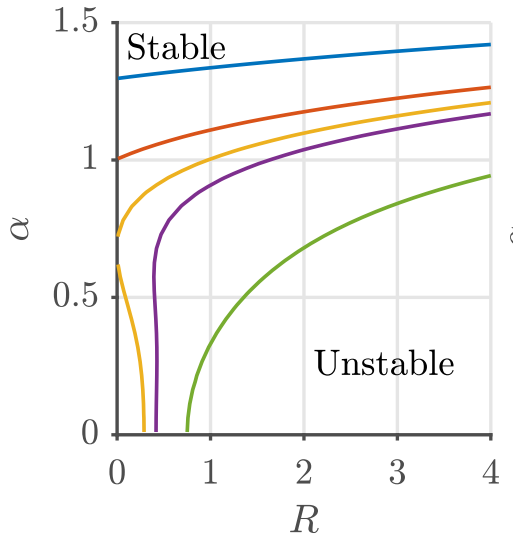

(a) $S=1$

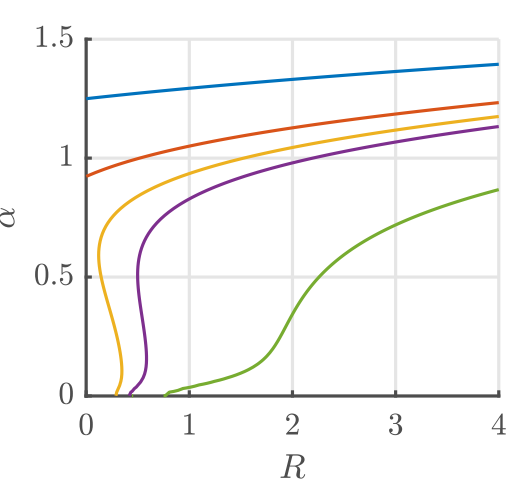

(b) $S=1000$

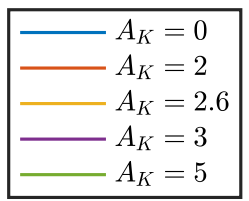

Figure 9: Neutral stability curves in the $\alpha-R$ plane as the wall stiffness varies (values of $A_{K}$ indicated on the plot), and for surface tension values $S=1$ - left, and $S=1000$ right. Other parameters are $\cot \beta=A_{D}=A_{T}=A_{B}=A_{I}=1$.

latter being more realistic for water. In both cases the rigid wall results begin to emerge for the largest value of $A_{K}=5$ (green curves), and we note the additional long wave destabilization for the $S=1000$ case by the flattening of the curve near the critical $R$. As $A_{K}$ increases further the flattened region disappears and a larger critical $R$ is found due to the larger $S$ involved. Figures $9 \mathrm{a}$ and $9 \mathrm{~b}$ also show that the finite wavenumber instabilities found analytically at $R=0$ are also supported when inertia is present. For both $S=1$ and $S=1000$ we observe islands of long wave stability when $R$ is small enough, e.g. the neutral curve for $A_{K}=2.6$ in Figure 9a. Larger $S$ stabilises the flow by shifting the neutral curves to the right to larger values of $R$ as seen in Figure $9 \mathrm{~b}$. If the stiffness is removed completely $\left(A_{K}=0\right.$, blue curve) the flow becomes unstable to long waves at all Reynolds numbers considered. For large $A_{K}$ the effect of the interfacial instability is dominant, while for small $A_{K}$ wall flexibility becomes more important thus explaining the slight effect of $S$ in this limit.

Corresponding growth rates from Figure 9a are presented in Figures 10a and 10b for $R=0.2$ and $R=3$, respectively, with the stiffness $A_{K}$ varying between 2.0 and 2.6. For $R=0.2$ we observe the long wave stabilization at the largest $A_{K}=2.6$ in line with the $R=0$ results of Figure 5b. As inertia increases to $R=3$, however, the long wave stabilization is lost and the band of unstable waves as well as the maximum growth rate, increase with $R$. At the same time the instability also increases with decreasing stiffness for fixed $R$, as seen in panel $10 \mathrm{~b}$.

Next, the effect of the wall damping $A_{D}$ is investigated for an inclination $\beta=\pi / 4$, all other parameters set to unity. Results are given in Figures 11a, 11b that show the neutral curves as $A_{D}$ varies, and representative growth rate curves at $R=1$. From the long-wave expression (4.3) we expect that for large $A_{D}$ the solution tends to the rigid wall limit, however it is not clear from the expansion how the limit is approached and we use computations to explore this. Two things are worth pointing out from the results of Figure 11a. First, for a given Reynolds number below the critical value for a rigid wall, we see that the damping does not fully stabilize the solution but supports a small band of unstable long waves - this can be seen clearly for $A_{D}=64$ in Figure 11a at Reynolds number of 1 and smaller, for example, and is in contrast to the large $A_{K}$ limit where stabilization occurs at a large but finite $A_{K}$ (e.g. Figure $9 \mathrm{a}$ for $A_{K}=5$ ). Second, 


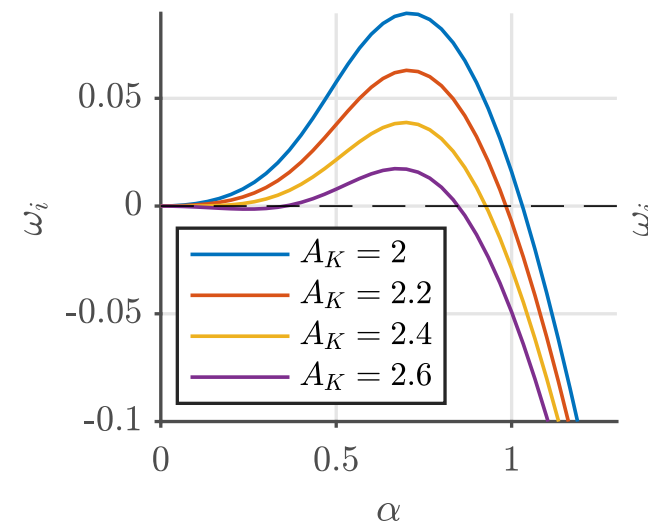

(a) $R=0.2$

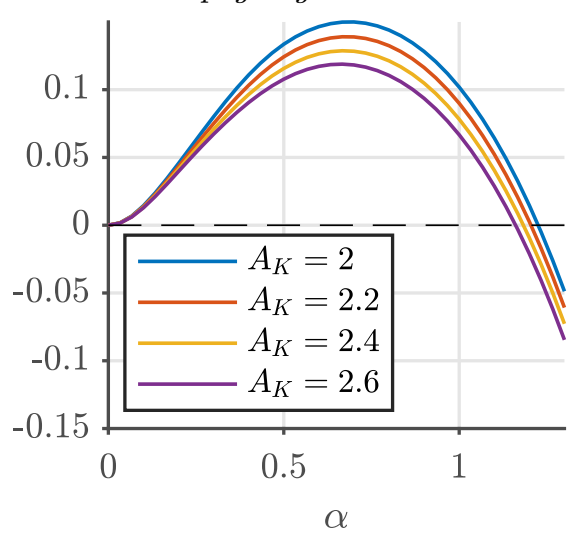

(b) $R=3$

Figure 10: Effect of inertia on the growth rates $\omega_{i}$ as $A_{K}$ varies (values shown on the plots): (a) $R=0.2$, (b) $R=3$. The other parameters are $\cot \beta=S=A_{D}=A_{T}=A_{B}=$ $A_{I}=1$.

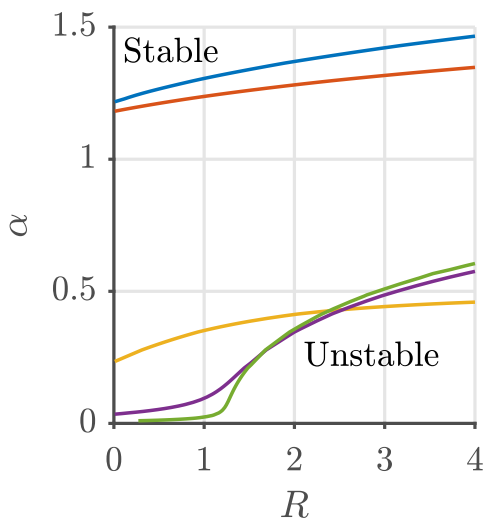

(a)
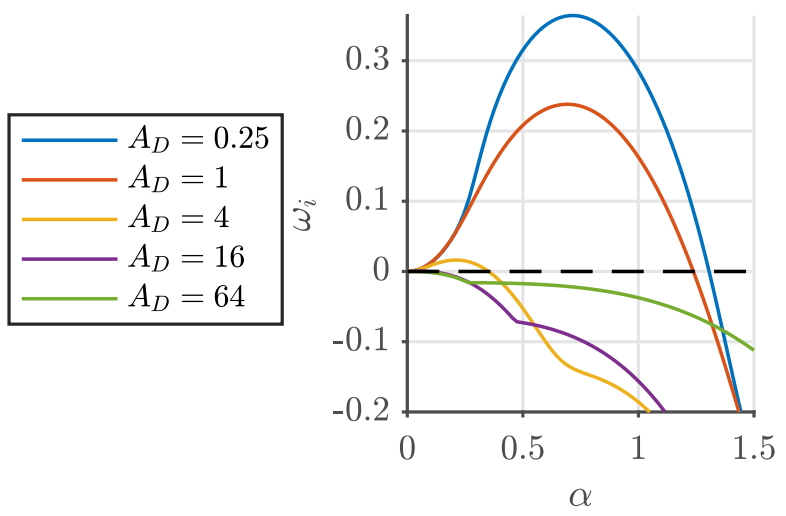

(b)

Figure 11: Stability characteristics as $A_{D}$ varies. (a) The neutral curves for the parameter set $\cot (\beta)=S=A_{T}=A_{B}=A_{K}=A_{I}=1$, and (b) the growth rate $\omega_{i}$ when $R=1$.

at larger $R$ damping is seen to have a stabilizing effect in the sense that it reduces the unstable region in the $\alpha-R$ plane; compare the cases $A_{D}=64$ with $A_{D}=4$ in Figure $11 \mathrm{a}$, for example, but at the same time note that this effect is non-monotonic in $A_{D}$ as seen from subsequent curves for $A_{D}=1$ and $A_{D}=0.25$. This phenomenon is due to fluid inertia and is not seen at either long waves or zero Reynolds numbers. Panel 11b shows the largest computed growth rate versus $\alpha$ for $R=1$; we observe that an increase in damping reduces the maximum growth rate as well as the neutral wavenumber. The results for $A_{D} \geqslant 4$ also indicate the presence of a second mode as found at zero Reynolds numbers in $\S 5$, Figure 6 for instance.

The results above show that long wave instability persists at large $A_{D}$ for the parameters chosen. Combined with the results of Figure 9a, we can surmise that this instability should in turn disappear as the stiffness $A_{K}$ is increased. To illustrate some of the characteristics and in particular to show underlying mechanisms such as mode crossings, we take $A_{D}=0.32, A_{K}=5$ and all other parameters equal to unity. At the 


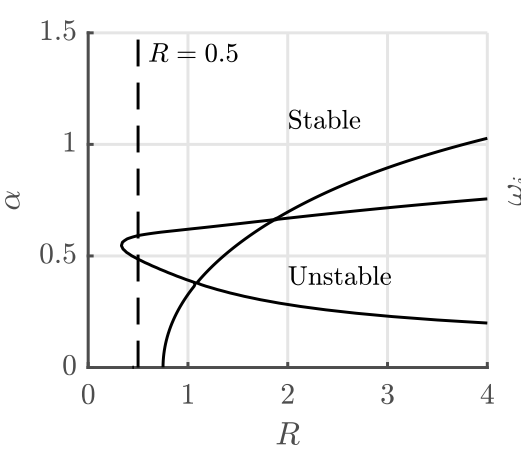

(a) $A_{D}=0.32$

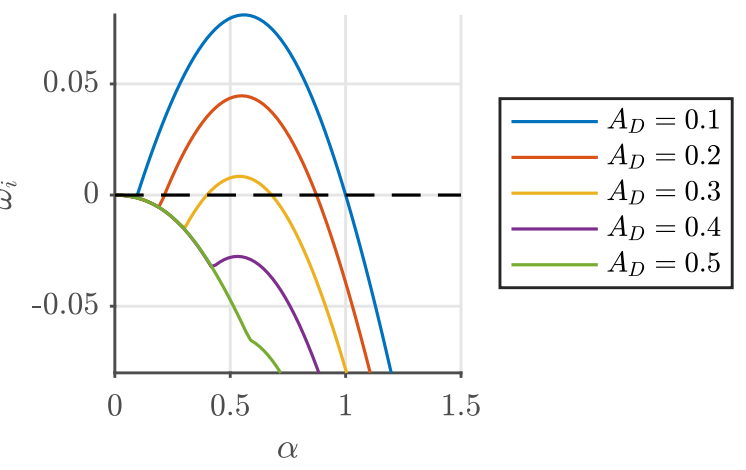

(b) $R=0.5$

Figure 12: (a) A neutral stability diagram; and (b) growth rate curves, each showing the presence of two unstable modes, for the parameter set: $A_{K}=5, \cot (\beta)=S=A_{T}=$ $A_{B}=A_{I}=1$. The growth rate curves also show the effect of increasing the damping $\left(A_{D}\right)$ on the two modes.

chosen parameter values we also find the coexistence of unstable interfacial and wall modes over a large range of wave numbers. Figure 12a shows the neutral curves in the $\alpha-R$ plane for the range $0 \leqslant R \leqslant 4$. We see that there are two modes present, the usual rigid wall interfacial mode with a critical Reynolds number for long waves, along with a second wall mode that has two branches for large $R$ (the upper branch grows with $R$ while the lower one decays as $R$ increases). An interesting finding from these results is that at small Reynolds numbers wall flexibility can destabilize the flow by reducing the critical Reynolds number and inducing a finite wavenumber instability. This can be seen from Figure 12a where the critical Reynolds number is reduced to approximately 0.332 and the first wavenumber that becomes unstable just above critical has $\alpha \approx 0.549$. For completeness in Figure $12 \mathrm{~b}$ we also present growth rates. Figure $12 \mathrm{~b}$ fixes $R=0.5$ (depicted by the vertical dashed line in Figure 12a), and shows the effect of varying $A_{D}$ between 0.1 and 0.5 on the most dominant mode. The presence of the two modes is seen in the plots and the finite wavenumber instability due to the wall mode is clear. The maximum growth rate increases as $A_{D}$ is decreased but the maximally unstable wavenumber varies weakly. We also note that at much higher $R$ there is another shear instability mode and the coexistence of all three modes and their relative growth rates are considered later.

\subsection{Instabilities at high Reynolds numbers}

It is well known in falling film flows that at higher Reynolds numbers shear modes of instability enter in addition to the interfacial mode that is prominent at lower values $R$ - see Floryan et al. (1987). Our model and computational tools enable us to carry out a detailed investigation of the effect of wall flexibility on such modes and indeed the interaction and competition between modes resulting from the three different physically supported instabilities. We will mostly present our results in the form of neutral stability curves in the $R-\alpha$ plane. Due to the large number of parameters, preliminary computations were carried out to identify which wall parameters are the most important, and in what follows we consider the effect of the stiffness parameter $A_{K}$ with a moderate amount of damping also present with $A_{D}=10$. The case computed has an inclination angle $\beta=4^{\circ}$ in order to extend the results of Floryan et al. (1987) to include wall flexibility. Representative results are given in figure 13 with the wall stiffness parameter 


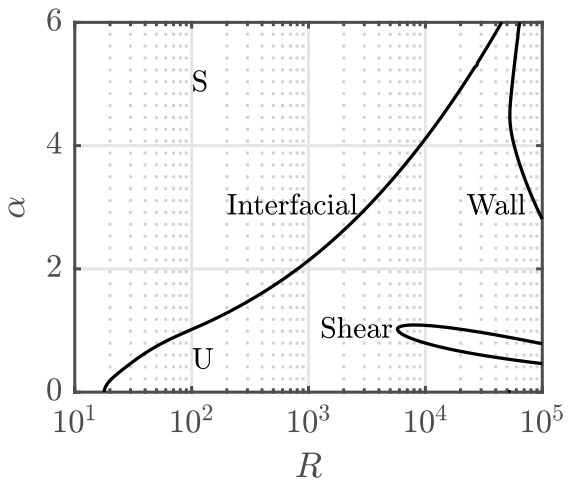

(a) $A_{K}=10^{5}$, (Almost rigid)

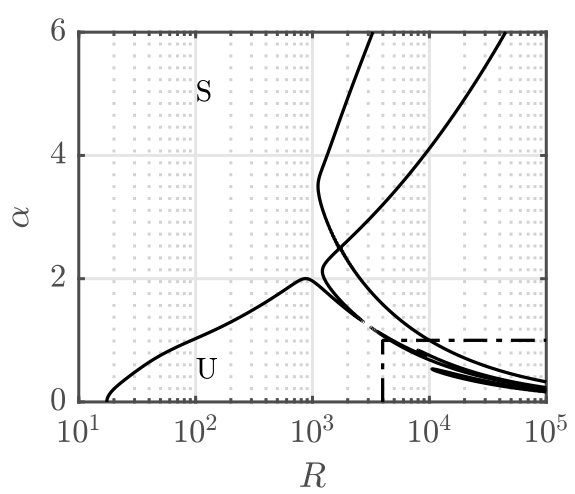

(c) $A_{K}=10^{3}$

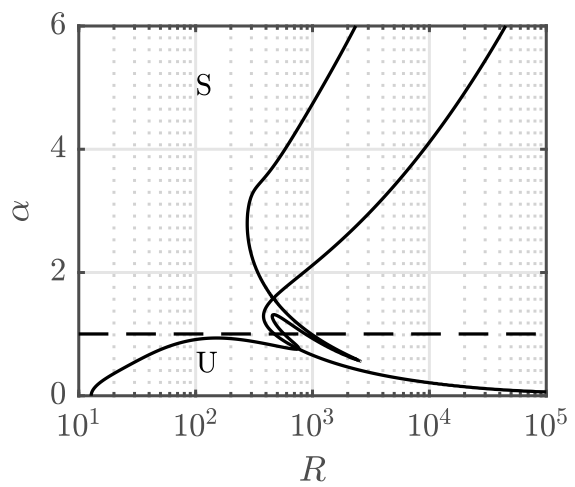

(e) $A_{K}=10^{2}$

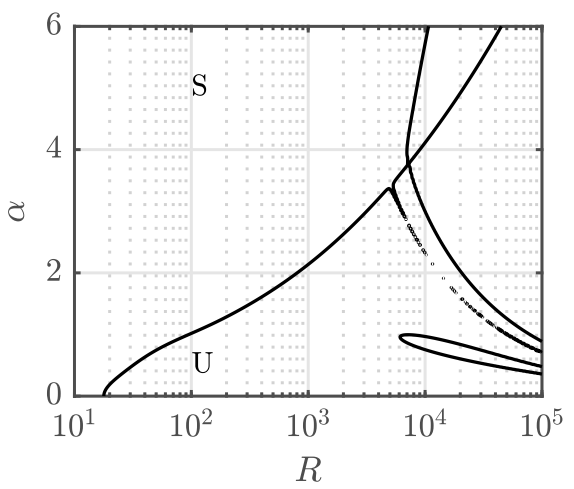

(b) $A_{K}=10^{4}$

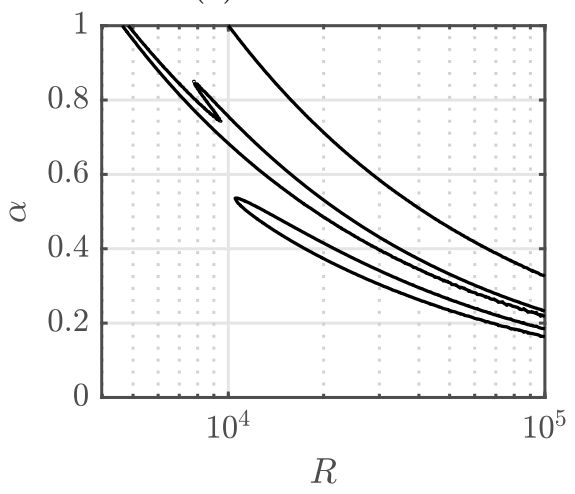

(d) $A_{K}=10^{3}$, Enlargement of dotted area in (c)

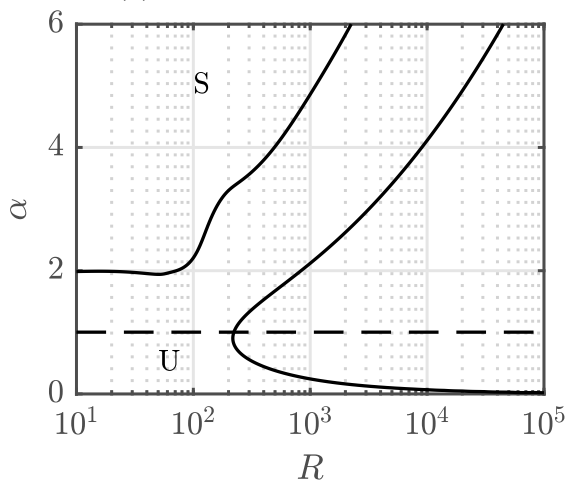

(f) $A_{K}=10$, (Highly flexible)

Figure 13: Neutral curves for the different modes in the $R-\alpha$ plane as the stiffness $A_{K}$ varies. The parameters are: $\beta=4^{\circ}, A_{D}=10$ with all remaining parameters set to 1 . The $\mathrm{U}$ and $\mathrm{S}$ labels refer to unstable and stable regions, respectively.

$A_{K}$ varying from $10^{5}$ in panel (a) to 10 in panel (f). All other wall parameters and surface tension are set to unity.

For large stiffness $A_{K}=10^{5}$, the wall is almost rigid. The results of Figure (13a) contain three different modes as labeled. These include the typical interfacial and shear mode neutral curves computed by Floryan et al. (1987), and in addition a mode due 
to wall flexibility; the first mode is a long wave interfacial one supporting instability at relatively small $R$, whereas the second is a shear mode that appears at larger $R \approx 5 \times 10^{3}$ - the shear mode was not seen in all previous results presented because the range of Reynolds numbers plotted was not large enough. The resulting plot is in full agreement with Floryan et al. (1987) once differences in non-dimensionalisation are accounted for. The wall mode enters at a critical $R \approx 5 \times 10^{4}$, and as $A_{K}$ increases this critical value also increases. It is described in more detail below for lower stiffnesses when it becomes more dominant.

Reducing the stiffness by an order of magnitude to $A_{K}=10^{4}$ produces the results of panel (13b). Several effects are notable: (i) the wall mode enters at a lower critical Reynolds number $R \approx 6 \times 10^{3}$, and enhances the band of unstable wavenumbers to include shorter waves at lower Reynolds numbers; (ii) the shear mode becomes a little more stable entering at a critical $R \approx 6 \times 10^{3}$, and at the same time narrowing its band of unstable wavenumbers; (iii) a break is observed in the interfacial mode with additional neutral curves appearing - this is due to an interaction between the different modes and is investigated fully for later plots by using an energy decomposition analysis.

The interaction of modes and the splitting of the interfacial mode found at large $A_{K}$, becomes clearer as $A_{K}$ decreases to $10^{3}$ as shown in Figure (13c). The wall mode becomes unstable at lower values of $R \approx 10^{3}$, and the intricate interaction behaviour in the lower right hand corner of the figure (identified by a dashed rectangular border) becomes clearer in the enlargement given in panel 13d. The shear mode is the lowest feature seen in the figure, and the flow is unstable everywhere. There are either one or two unstable modes in different parts of the depicted region. For example fixing $R=2 \times 10^{4}$ and varying $\alpha$, the number of unstable modes alternate between one and two every time a neutral curve is crossed.

In the last two panels $13 \mathrm{e}$ and $13 \mathrm{f}$ we follow the change in the neutral curves as $A_{K}$ is decreased to 100 and 10, respectively, the last case comprising the most flexible wall considered in this study. For $A_{K}=100$ the two main modes, namely interfacial and wall mode, are still prominent, the former at smaller $R$ and $\alpha$ and the latter at moderate $R$ and order one $\alpha$, but the results indicate further mode interaction and switching. For example, considering a fixed value $R=500$ as $\alpha$ is increased from zero we initially encounter instability due to the interfacial mode, followed by a small window of stability before additional modes enter at moderate $\alpha$, and eventually one wall mode remains at higher wavenumbers (complete stabilisation takes place at short waves due to surface tension). The presence of an interfacial mode at small $R$, coupled with a complete stability of the system at values of $R$ below a critical value, cease to exist for higher wall flexibility as illustrated in the results of panel $13 \mathrm{f}$ for $A_{K}=10$. In decreasing $A_{K}$ from 100 to 10, the merged interfacial and wall modes evolve to give the uppermost neutral curve, resulting in long-wave instability at small Reynolds numbers including $R=0$, consistent with Figure 5. At higher $R$ an additional mode enters with the flow remaining unstable everywhere to the right of the uppermost neutral curve that extends back to $R=0$. In panels 13e, 13f, the horizontal dashed lines drawn through $\alpha=1$ indicate the parameters over which we carry out an energy analysis that helps us identify the physical origin of the different instabilities as the Reynolds number is increased. This analysis and the results are presented next.

\subsection{Energy Analysis}

Due to the complexity of neutral stability plots such as those in Figure 13, we provide results of an energy decomposition of different terms in the perturbation equations in an effort to link particular modes with their physical origin. We proceed as in the energy 


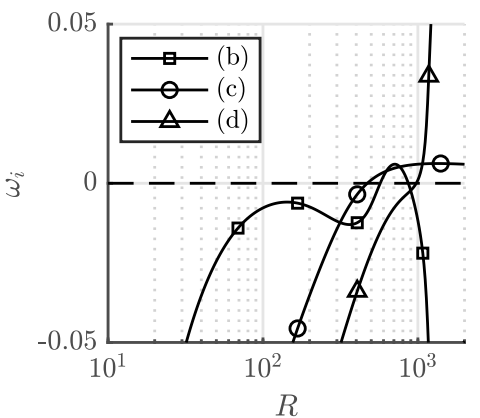

(a)

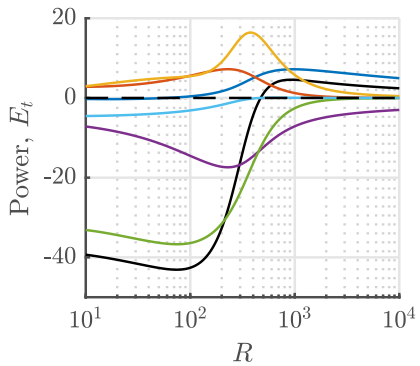

(c)

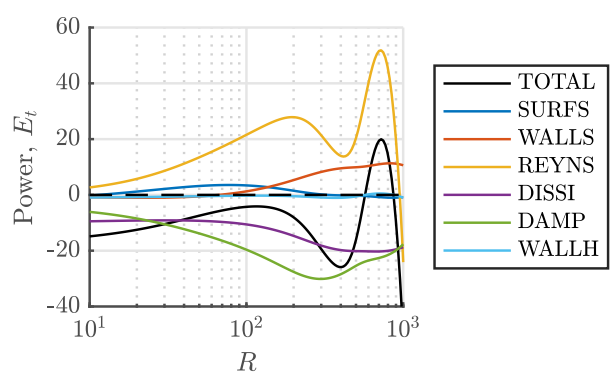

(b)

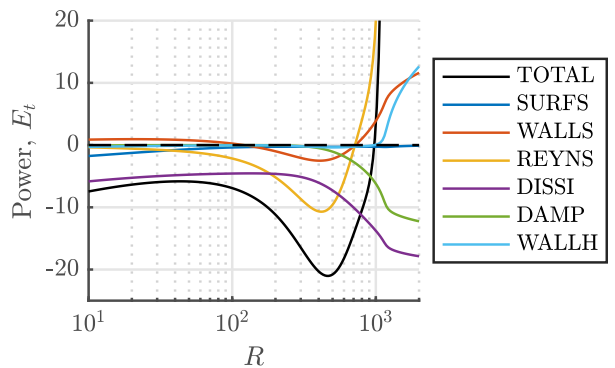

(d)

Figure 14: A growth rate plot (panel $(a)$ ) and the energy decompositions (panels $(b, c, d)$ ), see equation 6.3 , of the three unstable eigen-solutions for the case $\alpha=1.0026, \beta=4^{\circ}$, $A_{K}=100$ and $A_{D}=10$ with all other parameters set to unity.

decomposition analysis of Kelly et al. (1989) for rigid wall falling films. We start with the linearised equations for the perturbations in primitive variables and take inner products to derive an equation for the evolution of the kinetic energy of the system integrated over the periodic domain. The length of the period is taken to be $L=2 \pi / \alpha$, thus allowing direct comparison to the neutral curves. The surface and wall parameters enter the equation through the evaluation of integrals at the boundaries. Details of the derivation are given in Appendix B. The final result we need here is

$$
\frac{1}{2} \frac{\partial \mathcal{E}}{\partial t}=\mathrm{DISSI}+\mathrm{REYNS}+\mathrm{SURFS}+\mathrm{WALLS}+\mathrm{DAMP}+\mathrm{WALLH}
$$

where the energy $\mathcal{E} \geqslant 0$ is given by

$$
\begin{aligned}
\mathcal{E} & =E_{K I N}+E_{S U R F}+E_{W A L L}+E_{H Y D} \\
E_{K I N} & =R \iint_{\Omega}\left(u^{2}+v^{2}\right) d x d z \\
E_{S U R F} & =S \int_{-L / 2}^{L / 2} h_{x}^{2} d x \\
E_{W A L L} & =\int_{-L / 2}^{L / 2}\left(A_{I} \eta_{t}^{2}+A_{T} \eta_{x}^{2}+A_{B} \eta_{x x}^{2}+A_{K} \eta^{2}\right) d x \\
E_{H Y D} & =2 \cot \beta \int_{-L / 2}^{L / 2} h^{2} d x
\end{aligned}
$$




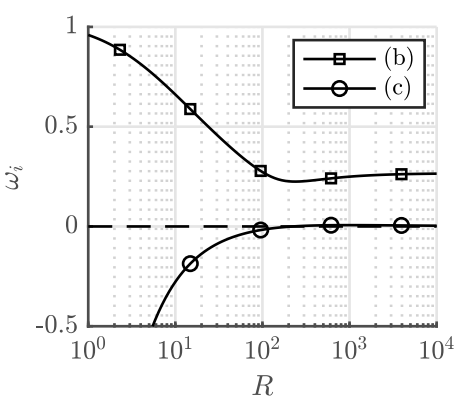

(a)

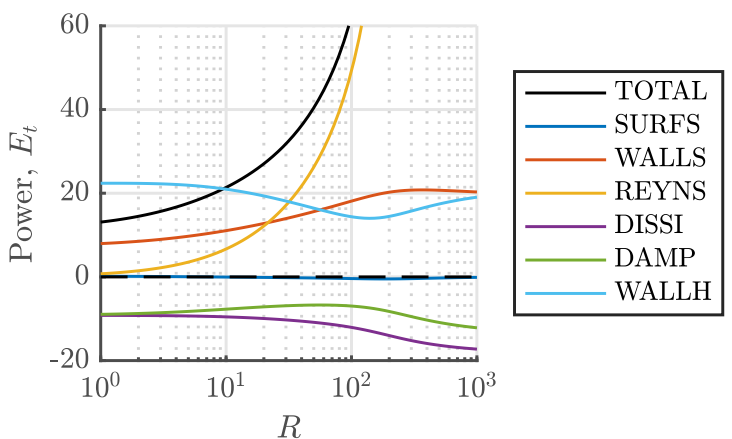

(b)

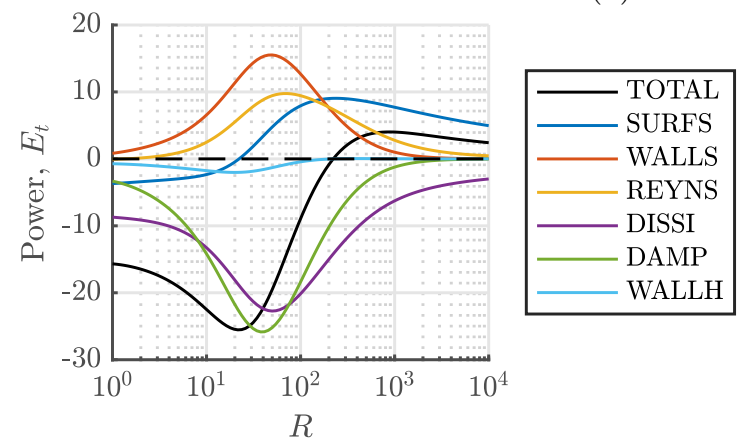

(c)

Figure 15: Plots showing the growth rate (panel $(a)$ ) and energy decompositions (panels $(b, c)$ ), see equation 6.3 , of the two unstable eigensolutions for the case: $\alpha=1.0026$, $\beta=4^{\circ}, A_{K}=10, A_{D}=10$ with all other parameters set to 1 .

where $E_{K I N}$ represents the kinetic energy within the fluid, $E_{S U R F}$ the energy stored in the deformed surface due to surface tension, $E_{W A L L}$ the energy contribution due to wall flexibility, and $E_{H Y D}$ denotes the hydrostatic potential energy. The right hand side includes the following terms that control the rate of change of the total energy

$$
\begin{aligned}
\text { DISSI } & =-\iint_{\Omega}\left(|\nabla u|^{2}+|\nabla v|^{2}\right) d x d z, \\
\text { REYNS } & =-2 R \iint_{\Omega} u v(1-z) d x d z, \\
\text { SURFS } & =\left.\int_{-L / 2}^{L / 2} u\left(u_{z}-v_{x}\right)\right|_{z=1} d x, \\
\text { WALLS } & =-\left.\int_{-L / 2}^{L / 2} u\left(u_{z}+v_{x}\right)\right|_{z=0} d x, \\
\text { DAMP } & =-A_{D} \int_{-L / 2}^{L / 2} \eta_{t}^{2} d x, \\
\text { WALLH } & =2 \cot (\beta) \int_{-L / 2}^{L / 2} \eta v d x .
\end{aligned}
$$

The viscous dissipation is denoted by DISSI and the Reynolds stress by REYNS. The rate of work done by shear on the surface and the wall enter as SURFS and WALLS, 
respectively. The damping term in the wall model contributes to the equation with the stabilising DAMP term and finally, WALLH, denotes a term originating from the work done by the hydrostatic pressure on the wall - see Appendix B.

Picking a wavenumber $\alpha$ and other system parameters, the linear stability results (eigenvalue-eigenfunction pairs) provide numerical values of each term on the right hand side of (6.3). In what follows we present results for $\alpha=1.0026$ and follow the instability as the Reynolds number increases along the dashed lines in figures $13 \mathrm{e}$ and 13f, with the corresponding energy decomposition results given in figures 14 and 15, respectively.

Results for $A_{K}=10^{2}$ are given in figure 14 for fixed $\alpha=1.0026$ as $R$ varies. Panel 14a shows the growth rates of the three most unstable modes for $R$ between 1 and $2 \times 10^{3}$ (we restricted the range of $R$ in order to make the results clearer). The modes are depicted by different symbols, with the corresponding energy decomposition of each mode provided in the accompanying panels as follows: mode 1 with square symbol is analyzed in panel 14b, mode 2 with circles is analyzed in panel 14c, and mode 3 with triangles in panel 14d. In the energy decomposition panels, each term from the RHS of the energy equation (6.3) is included by a different colour and identified in the legend. The black curve denoted by TOTAL provides the sum of all terms, i.e. the RHS of (6.3). As expected, the neutral Reynolds number predicted by this curve is identical to that predicted by the corresponding growth rate of each mode in panel 14a. In all cases the viscous dissipation (DISS) and wall damping (DAMP) are negative for all $R$ as expected, and play a significant role on the overall stability characteristics. At smaller $R$ they act to thwart growth due to other effects ensuring that a finite $R$ is needed for instability. Starting with mode 1 in panel $14 \mathrm{~b}$, we see that instability is mainly associated with Reynolds stresses, surface stresses and wall stresses. As these increase with $R$, viscous dissipation and wall damping also increase and delay the appearance of instability, that is in turn constrained to a small window in $R$ mainly due to the significant decrease of the Reynolds stresses from around $R \approx 7 \times 10^{2}$. We conclude that instability for this mode is due to Reynolds and wall stresses (yellow and red curves, respectively).

For mode 2 (circles), energy growth at smaller $R$ is dominated by Reynolds and wall stresses as seen in panel 14c. Other effects are stabilizing and in particular a sizeable viscous dissipation ensures negative growth rates. As $R$ increases to values between $10^{2}$ and $10^{3}$, Reynolds and wall stresses peak to maximum values before decaying significantly by $R \approx 10^{4}$. At the same time the surface shear stress term enters to provide energy growth, and coupled with weaker viscous dissipation and wall damping, an instability ensues as indicated in the figure. This instability is mainly due to the mechanism of energy growth due to surface shear stresses (SURFS, blue curve).

Mode 3 (triangular symbols) in panel 14d has quite different energy decomposition. At smaller $R$, energy growth is entirely due to wall stresses (red curve) but viscous dissipation dominates providing stability. As $R$ increases both wall stresses and Reynolds stresses decrease further to reach local negative minima, until $R \approx 7 \times 10^{2}$ when they both provide energy growth; in addition, the hydrostatic pressure wall mode (WALLH) produces energy growth beyond $R \approx 10^{3}$. As a result, and due to the large energy growth of the Reynolds stresses, the growth rate increases substantially as seen in panel 14a.

Finally we present results for the smallest value $A_{K}=10$ considered here. As in figure 14, we fix $\alpha=1.0026$ and look at the energetics of the dominant modes as $R$ is varied along the dashed line in figure 13f. This case is particularly interesting since instability is supported for all $R$, even $R=0$, unlike the other $A_{K}$ cases described. The presentation of the results in figure 15 follows that above. Only two unstable modes exist for $\alpha=1.0026$ and $0 \leqslant R \leqslant 10^{4}$, and their growth rates as $R$ varies are depicted in panel 15a. The energy decomposition of mode 1 (square symbols) is included in panel $15 \mathrm{~b}$ and 
the corresponding data for mode 2 (circles) appear in panel 15c. The results show that for small $R$ (including near zero) mode 1 is driven by the work done by the hydrostatic pressure on the wall (WALLH, the light blue curve) and the wall shear stresses (WALLS, red curve). At larger $R$ (higher than 25, approximately) the Reynolds stresses dominate while the effects of SURFS and WALLH level out to approximate equal values and so the physical mechanism responsible for instability switched from boundary effects to bulk Reynolds stresses. The surface shear stresses play no role in the dynamics explaining why this mode is not observable in the absence of wall elasticity. We also note that the viscous dissipation and wall damping terms suppress the instability but are not high enough to stabilize the flow at any values of $R$ considered. The energetics of mode 2 (circles in panel 15a) follow very similar trends to those of mode 2 of the larger $A_{K}=10^{2}$ case shown in figure $14 \mathrm{c}$, and so we can conclude that they are a continuation of one another and we do not need to discuss it further.

\section{Conclusions}

We carried out a linear stability study of falling liquid films over elastic substrates. A fairly general elastic wall model is used that has been studied in related problems - Carpenter \& Garrad (1985); Gajjar \& Sibanda (1996); Davies \& Carpenter (1997); Pruessner \& Smith (2015). We retain different physical elastic effects including wall inertia, damping, tension, flexural rigidity and stiffness and compute their effect on the stability of falling films. In the case of rigid walls, falling films become unstable to two modes of instability - a long wave interfacial (free surface) mode at lower Reynolds numbers (the critical Reynolds number tends to zero as the plate becomes vertical), and a higher Reynolds number shear mode or Tollmien-Schlichting mode - see Floryan et al. (1987). There are many dimensionless parameters in the problem - in addition to the five elasticity parameters and the Reynolds number, we have the inclination angle and the surface tension. We addressed the problem by using a combination of asymptotic analysis and full numerical computations of the elastically modified Orr-Sommerfeld problem based on the Chebyshev-Tau method. In order to understand the complex interaction between modes we also used an energy analysis to decompose them into constituent parts that originate from different physical mechanisms.

A long wave asymptotic analysis showed that the plate stiffness $A_{K}$ plays a crucial role in the dynamics. For example, the familiar rigid wall result of a critical Reynolds number $5(\cot \beta) / 4$ (in our non-dimensionalisation) below which the flow is stable to long waves, ceases to hold as plate stiffness decreases. In fact a critical $A_{K}$ is reached below which the flow is unstable to all Reynolds numbers including zero (see figure 3), a result that fully hinges on wall elasticity. Motivated by such results, we also carried out an analysis at zero $R$ which is valid for all wavenumbers. This limit is amenable to an analytical solution as given in Section 5. The results show that as the stiffness $A_{K}$ is reduced from large values (i.e. rigid wall and we $\operatorname{chose} \cot \beta=1$ so that when $A_{K} \rightarrow \infty$ the flow is stable for all wavenumbers), instability first enters at a finite wavenumber $\alpha$ prior to long waves becoming unstable - see figure 5. This result indicates that straightforward long wave theories are not appropriate for moderate values of $A_{K}$. The effect of wall damping $A_{D}$ was also considered starting with $A_{K}=1$ that ensures instability at zero $R$. Increasing $A_{D}$ from zero has a stabilising effect as expected (indeed as $A_{D} \rightarrow \infty$ we recover the rigid wall case), with the interfacial/surface mode providing the dominant instability. Interestingly as $A_{D}$ is decreased further we observe mode switching with the wall mode dominating the surface mode (see figure 7 , for example).

The asymptotic limits are useful and point to the need of the full numerical solution of 
the eigenvalue problem at arbitrary $R$ and $\alpha$. This was done for moderate as well as large $R$ in order to capture the interaction between the three modes present: surface, wall and shear modes. In the presence of wall elasticity we again find instability below the critical $R$ required for rigid walls. At moderate Reynolds numbers we again find instability below the critical $R$ due to the presence of the elastic wall as well as mode crossing between the surface and wall modes. The coexistence of surface and wall mode instabilities is ubiquitous in the presence of inertia, see for example figure $12 \mathrm{~b}$. The instability modes become much more complicated as $R$ is increased further. We studied the flow with an inclination angle $\beta=4^{\circ}$ in order to evaluate the effect of wall elasticity on the results of Floryan et al. (1987) for a rigid wall, and carried out computations to $R$ as large as $10^{6}$. The main parameter we studied in detail is the wall stiffness $A_{K}$ with other parameters set to unity. For $A_{K}=10^{5}$ the wall is almost rigid and the results are in agreement with Floryan et al. (1987) as shown in figure 13a; in addition to the surface and shear modes a wall mode enters at large $R$. As $A_{K}$ is reduced to values $10^{4}, 10^{3}, 10^{2}$ and 10 , the neutral stability curves become quite intricate due to wall elasticity and its effect on the eigenmodes and eigenvalues. Interesting instabilities arise, including mode splitting and switching as well as a destabilization of the flow for all $R$ when $A_{K}=10$ - see figure 13f. To understand such complex dynamics physically, we carried out an energy decomposition analysis and found that inertia drives the instabilities for larger $A_{K}$ (e.g. $A_{K}=100$ and higher), whereas at the smallest value studied $A_{K}=10$, at moderate Reynolds numbers the physical mechanism mostly responsible for the instability is the wall shear stress and the work done by the hydrostatic pressure on the wall - see figure 15 .

The present work has identified some instabilities that may be observable in practice and will hopefully motivate experiments in that direction. Of interest also would be nonlinear studies either via direct numerical simulations or using asymptotic theories. We note, however, that not all instabilities are long wave and hence lubrication type theories would not be appropriate, whereas weighted residual methods (see Kalliadasis et al. (2012)) could be a possible direction.

JPA acknowledges a PhD scholarship from the EPSRC. TLK was partly supported by an EPSRC Doctoral Prize Fellowship and DTP was partly supported by EPSRC grant number EP/L020564.

Declaration of Interests. The authors report no conflict of interest.

\section{Appendix A. Derivation of flexible wall model}

Even though we used Cartesian coordinates to describe the wall position $z=\eta(x, t)$, it is initially more convenient to use the parametrisation $\boldsymbol{X}(s, t)=(X(s, t), Z(s, t))$, where $s$ denoted arc length. (Both coordinate systems will be used interchangeably where convenient.) Newton's second law states

$$
\rho_{w} b \boldsymbol{X}_{t t}+D\left(\boldsymbol{e}_{z} \cdot \boldsymbol{X}_{t}\right) \boldsymbol{e}_{z}-\partial_{s}\left(T \boldsymbol{t}_{w}\right)+B \partial_{s s}\left(\kappa_{w} \boldsymbol{n}_{w}\right)+K\left(\boldsymbol{e}_{z} \cdot \boldsymbol{X}\right) \boldsymbol{e}_{z}=\left.\boldsymbol{T} \cdot \boldsymbol{n}_{w}\right|_{\boldsymbol{x}=\boldsymbol{X}}+p_{s} \boldsymbol{n}_{w},
$$

where $\boldsymbol{e}_{z}$ is the unit vector in the $z$ direction, and $\boldsymbol{t}_{w}$ and $\boldsymbol{n}_{w}$ are the unit tangent and normal to the plate (defined similarly to those for the liquid-gas interface, but with $h$ replaced with $\eta$; thus $\boldsymbol{n}_{w}$ points into the fluid), with $\kappa_{w}$ its curvature; $p_{s}$ is the substrate pressure on the back of the plate provided by the substrate fluid, its exact form and dependence on $x$ and $t$ also unknown, but assumed to be forced by the motion of the main flow and plate. Its exact evaluation will not be needed until we begin the linear stability analysis (§3), where its effect is much more easily assessed. 
The first term in (A 1) represents the inertia of the plate, the third term the plate's inextensibility, and the fourth term its rigidity. The inextensibility condition is $\partial_{s} \boldsymbol{X}$. $\partial_{s} \boldsymbol{X}=1$ for all $t$, i.e., that $s$ is indeed always the arc length, and thus $\boldsymbol{t}_{w}=\partial_{s} \boldsymbol{X}$ and $\kappa_{w} \boldsymbol{n}_{w}=\partial_{s s} \boldsymbol{X}$, allowing us to write the tension and rigidity terms in a more useful form,

$$
\begin{aligned}
\partial_{s}\left(T \boldsymbol{t}_{w}\right) & =\left(T \boldsymbol{X}_{s}\right)_{s}, \\
B \partial_{s s}\left(\kappa_{w} \boldsymbol{n}_{w}\right) & =B \boldsymbol{X}_{s s s s}
\end{aligned}
$$

(changing $\partial_{s}$ to subscript notation for brevity). The damping and stiffness terms are proportional to the projections of the plate velocity and plate displacement in the $z$ direction. Whether we choose to project in the $\boldsymbol{e}_{z}$ or $\boldsymbol{n}_{w}$ direction will not affect the resulting simplified wall equation.

We will now make the assumption of small deflections which (as well as a mechanical restriction of motion in the longitudinal direction owing to the presence of the springs) implies longitudinal deflections will be small compared to transverse deflections - Landau \& Lifshitz (1986). That is, we need only consider the normal (to the plate) component of the motion equation (A 1). This assumption has been used many times in the study of viscous fluid flows over flexible surfaces, such as Atabek \& Lew (1966); Dragon \& Grotberg (1991); Halpern \& Grotberg (1992) for flexible tubes, and Matar et al. (2007); Matar \& Kumar $(2004,2007)$ for flexible planes. The normal component is

$$
\begin{aligned}
\rho_{w} b \boldsymbol{X}_{t t} \cdot \boldsymbol{n}_{w} & +D\left(\boldsymbol{e}_{z} \cdot \boldsymbol{X}_{t}\right)\left(\boldsymbol{e}_{z} \cdot \boldsymbol{n}_{w}\right)-\left(T \boldsymbol{X}_{s}\right)_{s} \cdot \boldsymbol{n}_{w} \\
& +B \boldsymbol{X}_{s s s s} \cdot \boldsymbol{n}_{w}+K\left(\boldsymbol{e}_{z} \cdot \boldsymbol{X}\right)\left(\boldsymbol{e}_{z} \cdot \boldsymbol{n}_{w}\right)=\left.\boldsymbol{n}_{w} \cdot \boldsymbol{T} \cdot \boldsymbol{n}_{w}\right|_{\boldsymbol{x}=\boldsymbol{X}}+p_{s} .
\end{aligned}
$$

Next, we reparametrise the plate position as $(X(s, t), Z(s, t))=(x, \eta(x, t))$. Negligible longitudinal motion implies $X_{t} \ll Z_{t}$, i.e., $x$ is approximately constant in $t$ compared to $\eta$. Let $h_{0}$ be a characteristic film thickness, and $\delta$ be the characteristic size of deflections. The small deflection assumption means that the ratio $\epsilon=\delta / h_{0} \ll 1$, i.e., that the amplitude of deflections, $\eta$, will be much smaller than the film thickness. Under this assumption, $\eta_{x}=O(\epsilon) \ll 1$, the relations between $s$ and $x$ are

$$
\begin{aligned}
\mathrm{d} s & =\left(1+\eta_{x}^{2}\right)^{1 / 2} \mathrm{~d} x=\left(1+O\left(\epsilon^{2}\right)\right) \mathrm{d} x \\
\frac{\partial}{\partial s} & =\frac{1}{\left(1+\eta_{x}^{2}\right)^{1 / 2}} \frac{\partial}{\partial x}=\left(1+O\left(\epsilon^{2}\right)\right) \frac{\partial}{\partial x}
\end{aligned}
$$

and so the tension and rigidity terms (A 2) and (A 3) in terms of $x$ are

$$
\begin{aligned}
\left(T \boldsymbol{X}_{s}\right)_{s} & =\frac{1}{\left(1+\eta_{x}^{2}\right)^{1 / 2}} T_{x} \boldsymbol{t}_{w}+\frac{T \eta_{x x}}{\left(1+\eta_{x}^{2}\right)^{3 / 2}} \boldsymbol{n}_{w} \\
& =\left(1+O\left(\epsilon^{2}\right)\right) T_{x} \boldsymbol{t}_{w}+\left(1+O\left(\epsilon^{2}\right)\right) T \eta_{x x} \boldsymbol{n}_{w} \\
B \boldsymbol{X}_{s s s s} & =B\left(0, \eta_{x x x x}\right)+O\left(\epsilon^{2}\right)
\end{aligned}
$$

Substituting into the left hand side of (A 4) and neglecting $O\left(\epsilon^{2}\right)$ contributions in each term, we have

$$
\rho_{w} b \eta_{t t}+D \eta_{t}-T \eta_{x x}+B \eta_{x x x x}+K \eta=\left.\boldsymbol{n}_{w} \cdot \boldsymbol{T} \cdot \boldsymbol{n}_{w}\right|_{z=\eta}+p_{s}
$$

where we have used $\boldsymbol{n}_{w}=(0,1)+O(\epsilon)$ and the negligible longitudinal approximation $\boldsymbol{X}_{t} \approx\left(0, \eta_{t}\right), \boldsymbol{X}_{t t} \approx\left(0, \eta_{t t}\right)$. Although we have not specified the sizes of $u, v$ and $p$, we can still linearise the right hand side with respect to $\epsilon$, (equivalently with respect to $\eta_{x}$ ) 
to obtain

$$
\begin{aligned}
\left.\boldsymbol{n}_{w} \cdot \boldsymbol{T} \cdot \boldsymbol{n}_{w}\right|_{z=\eta}+p_{s} & =p_{s}-p+\frac{2 \mu}{\left(1+\eta_{x}^{2}\right)}\left(\left(1-\eta_{x}^{2}\right) v_{z}-\eta_{x}\left(u_{z}+v_{x}\right)\right) \\
& \approx p_{s}-p+2 \mu\left(v_{z}-\eta_{x}\left(u_{z}+v_{x}\right)\right) .
\end{aligned}
$$

Another condition on the fluid-substrate interface is the no-slip condition,

$$
\boldsymbol{u}=\boldsymbol{X}_{t}, \text { or }\left\{\begin{array}{l}
\boldsymbol{u} \cdot \boldsymbol{n}_{w}=\boldsymbol{X}_{t} \cdot \boldsymbol{n}_{w} \\
\boldsymbol{u} \cdot \boldsymbol{t}_{w}=\boldsymbol{X}_{t} \cdot \boldsymbol{t}_{w}
\end{array}\right.
$$

Since longitudinal motion is neglected, i.e., $\boldsymbol{X}_{t} \cdot \boldsymbol{t}_{w} \approx 0$, then $\boldsymbol{u} \cdot \boldsymbol{t}_{w}=\boldsymbol{X}_{t} \cdot \boldsymbol{t}_{w}$ implies $u+v \eta_{x} \approx 0$. If $v$ is no larger in size than $u$ (in fact it will be $O(\epsilon)$ smaller), then we can neglect $v \eta_{x}$ to get the condition

$$
u=0 \quad \text { at } \quad z=\eta .
$$

Similarly, $\boldsymbol{u} \cdot \boldsymbol{n}_{w}=\boldsymbol{X}_{t} \cdot \boldsymbol{n}_{w}$ implies $-\eta_{x} u+v=-\eta_{x} X_{t}+\eta_{t}$, but (A 9) and the assumption $X_{t} \ll \eta_{t}$ simplify this to

$$
v=\eta_{t} \quad \text { at } \quad z=\eta
$$

Thus, our three conditions on the plate, now in terms of $\eta$, are

$$
\begin{aligned}
v & =\eta_{t}, \\
u & =0 \\
\rho_{w} b \eta_{t t}+D \eta_{t}-T \eta_{x x}+B \eta_{x x x x}+K \eta & =p_{s}-p+2 \mu\left(v_{z}-\eta_{x}\left(u_{z}+v_{x}\right)\right),
\end{aligned}
$$

with all variables evaluated at $z=\eta$.

\section{Appendix B. Derivation of the energy equation}

The full linearised system in primitive variables are (dropping the tildes for perturbations introduced in Section 3)

$$
\begin{aligned}
R\left(u_{t}+\bar{u} u_{x}+v \bar{u}_{z}\right) & =-p_{x}+u_{x x}+u_{z z} \\
R\left(v_{t}+\bar{u} v_{x}\right) & =-p_{z}+v_{x x}+v_{z z}, \\
u_{x}+v_{z} & =0
\end{aligned}
$$

where $\bar{u}(z)=z(2-z)$. The boundary conditions are found by linearizing the kinematic, tangential stress and normal stress balance equations $(2.2 a),(2.2 b)$ and $(2.10)$ at the interface, and the no-slip and elastic substrate deflection equations (2.11a) and (2.11b) at the wall. Linearization is about the base-state (2.13). The equations are (their normal mode form is given in Section 3):

$\underline{z=1}$ :

$$
\begin{aligned}
& v=h_{t}+h_{x}, \\
& u_{z}+v_{x}+2 h=0, \\
& -p+2 v_{z}+2 \cot (\beta) h=S h_{x x} .
\end{aligned}
$$

$\underline{z=0}$ :

$$
\begin{aligned}
& u=-2 \eta \\
& v=\eta_{t}, \\
& A_{I} \eta_{t t}+A_{D} \eta_{t}-A_{T} \eta_{x x}+A_{B} \eta_{x x x x}+A_{K} \eta-2(\cot \beta) \eta=-p+2\left(v_{z}-2 \eta_{x}\right) .
\end{aligned}
$$


Multiplying (B 1) by $u$ and (B 2) by $v$ and adding, gives

$$
R\left(u u_{t}+u \bar{u} u_{x}+u \bar{u}_{z} v+v v_{t}+v \bar{u} v_{x}\right)=-\mathbf{u} \cdot \nabla p+\mathbf{u} \cdot \nabla^{2} \mathbf{u},
$$

where $\mathbf{u}=(u, v)$. The energy equation is found by integrating (B 10) over the domain $\Omega=\left[-\frac{L}{2}, \frac{L}{2}\right] \times[0,1]$, where $L$ is the wavelength of a periodic disturbance (i.e. $L=$ $2 \pi / \alpha)$ - we also denote the boundary of $\Omega$ by $\Gamma$. We follow Kelly et al. (1989) and also provide details due to the additional effects of wall elasticity. Integration by parts coupled with spatial periodicity immediately yields $\iint_{\Omega} \bar{u} u u_{x} d x d z=\int_{0}^{1} \bar{u}\left[\frac{1}{2} u^{2}\right]_{-\frac{L}{2}}^{\frac{L}{2}} d z=0$ and similarly $\iint_{\Omega} \bar{u} v v_{x} d x d z=0$, and hence the integral of (B 10) becomes

$$
\frac{\partial}{\partial t} \iint_{\Omega} \frac{1}{2} R\left(u^{2}+v^{2}\right) d x d z+\iint_{\Omega} 2 R(1-z) u v d x d z=\iint_{\Omega}\left(-\mathbf{u} \cdot \nabla p+\mathbf{u} \cdot \nabla^{2} \mathbf{u}\right) d x d z .
$$

On use of (B 3) the integrand of the first term on the right hand side (RHS) of (B 11) is $-\mathbf{u} \cdot \nabla p=-\nabla \cdot(p \mathbf{u})$, and by the divergence theorem we find

$$
\iint_{\Omega}(-\mathbf{u} \cdot \nabla p) d x d z=\left.\int_{-\frac{L}{2}}^{\frac{L}{2}}(p v)\right|_{z=0} d x-\left.\int_{-\frac{L}{2}}^{\frac{L}{2}}(p v)\right|_{z=1} d x .
$$

In arriving at (B 12) we note that the contributions at the boundaries $x= \pm L / 2$ cancel since periodicity implies $\left.\int_{0}^{1}(p u)\right|_{x=-\frac{L}{2}} d z=\left.\int_{0}^{1}(p u)\right|_{x=\frac{L}{2}} d z$. To compute the first integral on the RHS of (B 12) we use (B 9) to eliminate $p$ at $z=0$, and (B 8) to eliminate $v$ in favor of $\eta_{t}$ in almost all terms. The result is

$$
\begin{array}{r}
\left.\int_{-\frac{L}{2}}^{\frac{L}{2}}(p v)\right|_{z=0} \mathrm{dx}=-\frac{1}{2} \frac{\partial}{\partial t} \int_{-\frac{L}{2}}^{\frac{L}{2}}\left(A_{I} \eta_{t}^{2}+A_{T} \eta_{x}^{2}+A_{B} \eta_{x x}^{2}+\left(A_{K}-2 \cot (\beta)\right) \eta^{2}\right) d x \\
-A_{D} \int_{-\frac{L}{2}}^{\frac{L}{2}} \eta_{t}^{2} \mathrm{dx}+\left.2 \int_{-\frac{L}{2}}^{\frac{L}{2}} v\left(v_{z}-2 \eta_{x}\right)\right|_{z=0} \mathrm{dx} .
\end{array}
$$

From (B 7) we have $-2 \eta_{x}=u_{x}$ and hence the last term in (B 13) vanishes by conservation of mass. Next we consider the second integral on the RHS of (B 12) and now use the kinematic condition (B 4) and normal stress balance (B 6) to eliminate $p$ in favour of $h$ to find

$$
-\left.\int_{-\frac{L}{2}}^{\frac{L}{2}} p v\right|_{z=1} d x=-\frac{\partial}{\partial t} \int_{-\frac{L}{2}}^{\frac{L}{2}}\left(\frac{1}{2} S h_{x}^{2}+(\cot \beta) h^{2}\right) d x-\left.2 \int_{-\frac{L}{2}}^{\frac{L}{2}} v v_{z}\right|_{z=1} d x .
$$

Finally we consider the viscous term on the RHS of (B 11) and use integration by parts and periodicity to yield

$$
\iint_{\Omega} \mathbf{u} \cdot \nabla^{2} \mathbf{u} d x d z=-\iint_{\Omega}\left(|\nabla u|^{2}+|\nabla v|^{2}\right) d x d z+\int_{-\frac{L}{2}}^{\frac{L}{2}}\left(\left[u u_{z}\right]_{0}^{1}+\left[v v_{z}\right]_{0}^{1}\right) d x .
$$

With these results and defining

$$
\begin{gathered}
\mathcal{E}=R \iint_{\Omega}\left(u^{2}+v^{2}\right) d x d z+S \int_{-\frac{L}{2}}^{\frac{L}{2}} h_{x}^{2} d x \\
+\int_{-\frac{L}{2}}^{\frac{L}{2}}\left(A_{I} \eta_{t}^{2}+A_{T} \eta_{x}^{2}+A_{B} \eta_{x x}^{2}+A_{K} \eta^{2}\right) d x+2 \cot \beta \int_{-\frac{L}{2}}^{\frac{L}{2}} h^{2} d x
\end{gathered}
$$


equation (B 11) is re-arranged as

$$
\begin{array}{r}
\frac{1}{2} \frac{\partial \mathcal{E}}{\partial t}=-\iint_{\Omega}\left(|\nabla u|^{2}+|\nabla v|^{2}\right) d x d z-2 R \iint_{\Omega} u v(1-z) d x d z \\
-\left.2 \int_{-\frac{L}{2}}^{\frac{L}{2}} v v_{z}\right|_{z=1} d x+\int_{-\frac{L}{2}}^{\frac{L}{2}}\left(\left[u u_{z}\right]_{0}^{1}+\left[v v_{z}\right]_{0}^{1}\right) d x \\
-A_{D} \int_{-\frac{L}{2}}^{\frac{L}{2}} \eta_{t}^{2} d x+2 \cot \beta \int_{-\frac{L}{2}}^{\frac{L}{2}} \eta \eta_{t} d x
\end{array}
$$

The physical origin of the terms on the RHS of (B 17) are, in order of appearance, viscous dissipation and Reynolds stresses over the whole domain, work done by the shear at the interface and the wall, wall-damping inherent in the model, and work done by the wall against the hydrostatic pressure. The last term is replaced by $2 \cot \beta \int_{-\frac{L}{2}}^{\frac{L}{2}} \eta v d x$ using boundary condition (B 8). The final form (6.3) follows by rearranging the work done by the shear

$$
\begin{array}{r}
-\left.2 \int_{-\frac{L}{2}}^{\frac{L}{2}} v v_{z}\right|_{z=1} d x+\int_{-\frac{L}{2}}^{\frac{L}{2}}\left(\left[u u_{z}\right]_{0}^{1}+\left[v v_{z}\right]_{0}^{1}\right) d x \\
=\int_{-\frac{L}{2}}^{\frac{L}{2}}\left[\left.\left(u u_{z}-v v_{z}\right)\right|_{z=1}-\left.\left(u u_{z}+v v_{z}\right)\right|_{z=0}\right] d x \\
=\left.\int_{-\frac{L}{2}}^{\frac{L}{2}} u\left(u_{z}-v_{x}\right)\right|_{z=1} d x-\left.\int_{-\frac{L}{2}}^{\frac{L}{2}} u\left(u_{z}+v_{x}\right)\right|_{z=0} d x
\end{array}
$$

and these are the terms SURFS and WALLS appearing in (6.3).

The final step is the calculation of the various terms in (6.3) using the computed eigensolutions. We illustrate this for the Reynolds stress term REYNS. Introducing the normal mode solutions of Section 3, and using superscripts $*$ to denote complex conjugates, we have

$$
\begin{aligned}
& \text { REYNS }=-2 R \iint_{\Omega}(1-z) u v d x d z \\
& =2 R \iint_{\Omega}(1-z)\left(\phi_{z} e^{i \alpha(x-c t)}+\phi_{z}^{*} e^{-i \alpha\left(x-c^{*} t\right)}\right)\left(i \alpha \phi e^{i \alpha(x-c t)}-i \alpha \phi^{*} e^{-i \alpha\left(x-c^{*} t\right)}\right) d x d z \\
& =4 i \pi \operatorname{Re}^{2 \alpha c_{i} t} \int_{0}^{1}(1-z)\left(\phi \phi_{z}^{*}-\phi^{*} \phi_{z}\right) d z \\
& =-8 \pi \operatorname{Re}^{2 \alpha c_{i} t} \int_{0}^{1}(1-z) \mathfrak{I}\left[\phi \phi_{z}^{*}\right] d z,
\end{aligned}
$$

where $\mathfrak{I}$ denoted the imaginary part of a quantity. Analogous calculations hold for all other terms, enabling a numerical computation of contributing terms.

\section{REFERENCES}

AtABek, H. B. \& Lew, S. H. 1966 Wave propagation through a viscous incompressible fluid contained in an initially stressed elastic tube. Biophys. J. 6, 481-503.

Baingne, M. \& Sharma, G. 2019 Effect of wall deformability on the stability of surfactantladen visco-elastic liquid film falling down an inclined plane. J. Non-Newtonian Fluid Mech. 269, 1-16.

Baingne, M. \& Sharma, G. 2020 Effect of wall deformability on the stability of shear-imposed film flow past an inclined plane. Int. J. Eng. Sci. 150, 103243. 
Benjamin, T. B. 1957 Wave formation in laminar flow down an inclined plane. J. Fluid Mech. 2, 554-574.

Benney, D. J. 1966 Long waves on liquid films. J. Math. Phys. 45, 150-155.

BINNy, A. M. 1957 Experiments on the onset of wave formation on a film of water flowing down a vertical plane. J. Fluid Mech. 2, 551-555.

Carpenter, P. W. \& Garrad, A. D. 1985 The hydrodynamic stability of flow over Kramertype compliant surfaces. Part 1 Tollmien-Schlichting instabilities. J. Fluid Mech. 155, $465-510$.

Chokshi, P. \& Kumaran, V. 2008 Weakly nonlinear stability analysis of a flow past a neoHookean solid at arbitrary Reynolds numbers. Phys. Fluids 20, 094109.

Davies, C.J. \& CARPenter, P.W. 1997 Instabilities in a plane channel flow between compliant walls. J. Fluid Mech. 352, 205-243.

Dawkins, P. T., Dunbar, S. R. \& Douglass, R. W. 1998 The origin and nature of spurious eigenvalues in the spectral tau method. J. Comput. Phys. 147, 441-462.

Dongarra, J. J., Straughan, B. \& Walker, D. W. 1996 Chebyshev tau-QZ algorithm methods for calculating spectra of hydrodynamic stability problems. Appl. Numer. Math. 22 (4), 399-434.

Dragon, C. A. \& Grotberg, J. B. 1991 Oscillatory flow and mass transport in a flexible tube. J. Fluid Mech. 231, 135-155.

Floryan, J.M., Davis, S.H. \& Kelly, R.E. 1987 Instabilities of a liquid film flowing down a slightly inclined plane. Phys. Fluids 30, 983-989.

GajJAR, J.S.B. \& SibandA, P. 1996 The hydrodynamic stability of channel flow with compliant boundaries. Theor. Comput. Fluid Dyn. 8, 105-129.

Gaurav \& Shankar, V. 2007 Stability of gravity-driven free-surface flow past a deformable solid at zero and finite Reynolds number. Phys. Fluids 19.

Gaurav \& Shankar, V. 2010 Role of wall deformability on interfacial instabilities in gravitydriven two-layer flow with a free surface. Phys. Fluids 22.

GKanis, V. \& Kumar, S. 2006 Instability of gravity-driven free-surface flow past a deformable elastic solid. Phys. Fluids 18.

Halpern, D. \& Grotberg, J. B. 1992 Fluid-elastic instabilities of liquid-lined tubes. J. Fluid Mech. 244, 615-632.

Jain, A. \& Shankar, V. 2007 Instability suppression in viscoelastic film flows down an inclined plane lined with a deformable solid layer. Phys. Rev. E. 76, 046314.

Kalliadasis, S., Ruyer-Quil, C., Scheid, B. \& Velarde, M.G. 2012 Falling Liquid Films. Springer-Verlag, London.

KAPITZA, P. L. \& KAPITZA, S. P. 1949 Wave flow of thin viscous liquid films. III. Experimental study of wave regime of a flow. J. Exp. Theor. Phys. 19, 105-120.

Kelly, R E, Goussis, D A, Lin, S P \& Hsu, F K 1989 The mechanism for surface wave instability in film flow down an inclined plane. Physics of Fluids A: Fluid Dynamics 1 (5), 819-828.

Kramer, M. O. 1957 Boundary-layer stabilisation by distributed damping. J. Aero. Sci. 24, 459.

Landau, L. D. \& Lifshitz, E. M. 1986 Theory of Elasticity. Butterworth-Heinemann.

Liu, J., Schneider, J. B. \& Gollub, J. P. 1995 Three-dimensional instabilities of film flows. Phys. Fluids 7 (1), 55-67.

Mandloi, S. \& Shankar, V. 2020 Stability of gravity-driven free-surface flow past a deformable solid: The role of depth-dependent modulus. Phys. Rev. E 101.

Matar, O. K., Craster, R. V. \& Kumar, S. 2007 Falling films on flexible inclines. Phys. Rev. E 76 (056301).

Matar, O. K. \& Kumar, S. 2004 Rupture of a surfactant-covered thin liquid film on a flexible wall. SIAM J. Appl. Math. 64 (6), 2144-2166.

Matar, O. K. \& Kumar, S. 2007 Dynamics and stability of flow down a flexible incline. J. Eng. Math. 57 (2), 145-158.

McFadden, G. B., Murray, B. T. \& Biosvert, R.F. 1990 Elimination of spurious eigenvalues in the Chebyshev tau spectral method. J. Comput. Phys. 91, 228-239.

Patne, R. \& Shankar, V. 2019 Stability of flow through deformable channels and tubes: implications of consistent formulation. J. Fluid Mech. 860, 837-885. 
Peng, J., Jiang, L. Y., Zhuge, W. L. \& Zhang, Y. J. 2016 Falling film on a flexible wall in the presence of insoluble surfactant. J. Eng. Math. 97, 33-48.

Peng, J., Zhang, Y. J. \& Zhuge, W. L. 2014 Falling film on a flexible wall in the limit of weak viscoelasticity. J. Non-Newton. Fluid 210, 85-95.

Pruessner, L. \& Smith, F.T. 2015 Enhanced effects from tiny flexible in-wall blips and shear flow. J. Fluid Mech. 772, 16-41.

Ruyer-Quil, C. \& Manneville, P. 2000 Improved modeling of flows down inclined planes. Eur. Phys. J. B 15, 357-369.

Sahu, S. \& Shankar, V. 2016 Passive manipulation of free-surface instability by deformable solid bilayers. Phys. Rev. E 94.

Shankar, V. \& Sahu, Akilesh K. 2006 Suppression of instability in liquid flow down an inclined plane by a deformable solid layer. Phys. Rev. E 73 (016301).

SHKAdOV, VY. 1967 Wave flow regimes of a thin layer of a viscous liquid under the action of gravity. Fluid Dyn. 2, 29-34.

Sisoev, G.M., Matar, O. K., Craster, R. V. \& Kumar, S. 2010 Coherent wave structures on falling fluid films flowing down a flexible wall. Chem. Eng. Sci. 65, 950-961.

Sivashinsky, G. I. \& Michelson, D. M. 1980 On irregular wavy flow on liquid film down a vertical plane. Prog. Theor. Phys. 63, 2112-2114.

Thaokar, R.M., Shankar, V. \& Kumaran, V. 2001 Effect of tangential interface motion on the viscous instability in fluid flow past flexible surfaces. Eur. Phys. J. B 23, 533-550.

Tseluiko, D. \& Papageorgiou, D. T. 2006 Wave evolution on electrified falling films. J. Fluid Mech. 556, 361-386.

Whiтнам, G.B. 1999 Linear and Nonlinear Waves. John Wiley \& Sons.

Yıн, C.-S. 1963 Stability of liquid flow down an inclined plane. Phys. Fluids 6 (3), 321-334. 